\title{
EQUIVALENCE OF MARKOV PROCESSES $\left({ }^{1}\right)$
}

\author{
BY \\ DONALD A. DAWSON
}

1. Introduction. The question of the measure theoretic equivalence of one stochastic process with respect to another has been studied in several contexts. For example, A. V. Skorokhod [16], [17] has considered the equivalence of Markov processes defined by means of stochastic differential equations, L. A. Shepp [15] has obtained criteria for the equivalence of Gaussian processes and the Wiener process, and E. B. Dynkin [7] has obtained a global criterion for the absolute continuity of one Markov process with respect to another in terms of multiplicative functionals.

In this paper we investigate the equivalence of Hunt processes from a local point of view. After introducing the basic notation and definitions in $\S 2$ we discuss three special cases in $\$ 3$ which motivate and illustrate the general theory which is to be developed. In $\$ 4$ a class of martingales is associated with a Markov process; this leads to the proof of an extended Markov property in $\S 5$. The extended Markov property is central in proving our results on the equivalence of Hunt processes. $\S \S 6$ and 7 are concerned with certain technical results which lead to the statement and proof of the main results in $\S \S 8$ and 9. In $\S 8$ it is shown that if two Hunt processes arising from Feller semigroups are not equivalent then at least one of four basic types of singularity must occur. The four basic types of singularity are: singularity on the germ field, singularity on the tail field, local singularity at a stopping time and jump singularity at a stopping time. $\$ 9$ contains a deeper study of local singularities. In particular, if the processes have the additional property which we call local smoothness then the existence of local singularities at a stopping time is equivalent to the existence of singularities on the germ field. Finally, in $\$ 10$ the property of local smoothness is characterized in potential theoretic terms.

The author would like to thank Professer P. A. Meyer and an anonymous referee for several very useful comments.

2. Notation and definitions. In this section we introduce the basic notation and review the important definitions. For a more complete discussion of the standard material refer to E. B. Dynkin [7] and P. A. Meyer [13]. A summary of the basic definitions in the theory of Markov processes is also found in R. K. Getoor [8]. Basic results of measure theory are often used without explicit reference; such material is found in the basic texts of J. Neveu [14] and P. R. Halmos [9].

Received by the editors August 1, 1966 and, in revised form, December 15, 1966.

(1) This research was supported in part by the Summer Research Institute of the Canadian Mathematical Congress. 
2.1. The state space. Let $(E, \mathscr{E})$ be a measurable space such that $E$ is a locally compact separable metric space with metric $\rho(\cdot, \cdot)$ and $\mathscr{E}$ is the $\sigma$-field of Borel subsets of $E$. Note that each open subset and each closed subset in such a space is a countable union of compact sets. Hence every set in the algebra generated by the open (or compact) subsets is the countable union of compacts. Moreover all finite Borel measures are inner regular. Let $\Delta$ be the point at infinity if $E$ is not compact or an isolated point if $E$ is compact. Let $E_{\Delta}=E \cup\{\Delta\}$ and $\mathscr{E}_{\Delta}$ be the $\sigma$-field of Borel subsets of $E_{\Delta}$.

2.2. The basic measure space. Let $\Omega$ be the set of all functions, $\omega(\cdot)$, from $[0, \infty]$ to $E_{\Delta}$ which are right continuous, have left-hand limits on $[0, \infty)$, have the property that if $\omega(t)=\Delta$, then $\omega(s)=\Delta$ for all $s \geqq t$, and satisfy $\omega(\infty)=\Delta . \omega_{\Delta}$ represents the element of $\Omega$ defined by $\omega_{\Delta}(t)=\Delta$ for all $t \in[0, \infty]$. If $\omega \in \Omega$, the mapping $t \leadsto X_{t}(\omega)$ $=X(t, \omega) \equiv \omega(t)$ is called the path of $\omega$. For $t \in[0, \infty), X_{t}^{-}(\omega)=\lim _{s} \lambda_{t} X_{s}(\omega)$. For $t \in[0, \infty]$ let $\mathscr{F}_{t}$ be the $\sigma$-field of subsets of $\Omega$ generated by sets of the form $\left\{\omega: X_{s}(\omega) \in \Gamma\right\}$ with $s \in[0, t]$ and $\Gamma \in \mathscr{E}_{\Delta}$ and for $t \in[0, \infty)$ let $\mathscr{M}_{t} \equiv \bigcap_{s>t} \mathscr{F}_{s}$, $\mathscr{M}_{\infty} \equiv \mathscr{F}_{\infty} . \mathscr{M}_{0}$ is known as the germ field.

Proposition 2.2.1. For any $s \in[0, \infty], \mathscr{F}_{s}$ is generated by a countable algebra of sets.

Proof. Let $\mathscr{A}_{s}$ be the algebra of subsets of $E$ generated by sets of the form $\left\{\omega: X_{r}(\omega) \in \Gamma_{j}\right\}$ with $r$ a rational in $[0, s]$ and $\Gamma_{j}$ an element of the countable base $\left\{\Gamma_{j}: j=1,2,3, \ldots\right\}$ of open sets for $E$. It suffices to show that a set of the form $\left\{\omega: X_{r}(\omega) \in \Gamma\right\}$ with $r$ an irrational number in $[0, s]$ and $\Gamma$ an open subset of $E$ is contained in $\sigma\left(\mathscr{A}_{s}\right)$, the $\sigma$-field generated by $\mathscr{A}_{s}$. But the right continuity of the paths implies that

$$
\left\{\omega: X_{r}(\omega) \in \Gamma\right\}=\bigcup_{j \in \mathscr{J}} \bigcup_{n=1}^{\infty} \bigcap_{k=n}^{\infty}\left\{\omega: X_{r_{n}}(\omega) \in \Gamma_{j}\right\}
$$

where $\left\{r_{n}\right\}$ is a sequence of rationals which decrease to $r$ and $\mathscr{J} \equiv\left\{j: \bar{\Gamma}_{j} \subset \Gamma\right\}$. Since $\bigcup_{j \in \mathscr{J}} \bigcup_{n=1}^{\infty} \bigcap_{k=n}^{\infty}\left\{\omega: X_{r_{n}}(\omega) \in \Gamma_{j}\right\} \in \sigma\left(\mathscr{A}_{s}\right)$, the proof is complete.

A mapping $T: \Omega \rightarrow[0, \infty]$ is a stopping time with respect to the $\sigma$-fields $\left(\mathscr{M}_{t}\right)_{t \in[0, \infty]}$ if for every $t \in[0, \infty],\{T<t\} \in \mathscr{M}_{t}$. If $T$ is a stopping time, then $\mathscr{M}_{T}$ denotes the sub- $\sigma$-field of sets $A \in \mathscr{F}_{\infty}$ such that $A \cap\{T \leqq t\} \in \mathscr{M}_{t}$ for every $t \in[0, \infty]$.

If $\zeta(\omega) \equiv \inf \left\{t: X_{t}(\omega)=\Delta\right\}$, then $\zeta$ is a stopping time and is called the lifetime of the path.

If the mapping $Y(\cdot, \cdot):\left([0, t] \times \Omega, \mathscr{B}_{[0, t]} \times \mathscr{M}_{t}\right) \rightarrow(F, \mathscr{F})$ where $(F, \mathscr{F})$ is a measurable space and $\mathscr{B}_{[0, t]}$ is the $\sigma$-field of Borel subsets of $[0, t]$ is measurable for every $t \in[0, \infty)$, then the stochastic process $Y(\cdot, \cdot)$ is said to be progressively measurable.

Proposition 2.2.2. (i) $X_{s}(\omega)$ is a progressively measurable stochastic process.

(ii) If $T$ is a stopping time, then $X_{T}$ is $\mathscr{M}_{T}$-measurable.

Proof. See P. A. Meyer [13, Chapter 4, §3]. 
2.3. Definition of a Markov process. The system $\pi=\left(\Omega, \mathscr{F}_{\infty},\left(P_{x}\right)_{x \in E_{\Delta}},\left(X_{t}\right)_{t \in[0, \infty]}\right)$ is a strong Markov process with respect to the $\sigma$-fields $\left(\mathscr{M}_{t}\right)_{t \in[0, \infty]}$ if:

(2.3.1) $P_{x}\left(X_{0}=x\right)=1$ for each $x \in E_{\Delta}$,

(2.3.2) the mapping $x \leadsto P_{x}(A)$ is $\mathscr{E}_{\Delta}$-measurable for $A \in \mathscr{F}_{\infty}$, and

(2.3.3) for any stopping time $T, \Gamma \in \mathscr{E}_{\Delta}$ and $s \in[0, \infty)$,

$$
P_{x}\left(X_{T+s} \in \Gamma \mid \mathscr{M}_{T}\right)=P_{X(T)}\left(X_{s} \in \Gamma\right),
$$

$P_{x}$-almost surely for each $x \in E_{\Delta}$.

$E_{x}$ denotes the expectation with respect to the measure $P_{x}$. The Markov process is said to be continuous if the paths are continuous on $[0, \zeta), P_{x}$-almost surely for each $x \in E$. It is said to be uninterrupted if for every $x \in E, P_{x}(\zeta=\infty)=1$.

2.4. The semigroup. Given an $\mathscr{E}_{\Delta}$-measurable bounded function, $f$, let

$$
P_{t} f(x) \equiv \int f(y) P_{x}\left(X_{t} \in d y\right)
$$

$C_{0}\left(E_{\Delta}\right)$ designates the set of continuous functions, $f$, on $E_{\Delta}$ such that $\lim _{y \rightarrow \Delta} f(y)=0$ and $f(\Delta)=0$. The semigroup $\left\{P_{t}: t \geqq 0\right\}$ is a Feller semigroup if:

(2.4.1) for every function $f \in C_{0}\left(E_{\Delta}\right)$ and $t>0, P_{t} f \in C_{0}\left(E_{\Delta}\right)$, and

(2.4.2) for every function $f \in C_{0}\left(E_{\Delta}\right),\left\|P_{t} f-f\right\| \rightarrow 0$ as $t \rightarrow 0$ where

$$
\|g\| \equiv \sup \{|g(x)|: x \in E\} .
$$

The following result is well known.

Proposition 2.4.1. If $\left\{P_{t}: t \geqq 0\right\}$ is a Feller semigroup, then

(i) if $f \in C_{0}\left(E_{\Delta}\right), P_{t} f(x)$ is jointly continuous in t and $x$ on $[0, \infty) \times E_{\Delta}$, and

(ii) $P_{x}(\zeta=0)=0$ for each $x \in E$.

2.5. Definition of a Hunt process. The strong Markov process $\pi$ is said to be quasi-left continuous if for any increasing sequence of stopping times $\left\{T_{n}\right\}$ with limit $T, X\left(T_{n}\right) \rightarrow X(T), P_{x}$-almost surely on $\{T<\infty\}$ for each $x \in E$. A strong Markov process as described in 2.3 which is quasi-left continuous is known as a Hunt process. In the remainder of this paper the term Hunt process refers to a quasi-left continuous strong Markov process in the canonical form described in 2.2 and 2.3 with a Feller semigroup.

2.6. The translation operators. For $t \in[0, \infty]$ the translation operator $\theta_{t}: \Omega \rightarrow \Omega$ is defined by

$$
\begin{aligned}
\left(\theta_{t} \omega\right)(s) & =\omega(s+t), \quad t<\infty, \\
\theta_{\infty} \omega & =\omega_{\Delta} \text { for every } \omega .
\end{aligned}
$$

If $T: \Omega \rightarrow[0, \infty]$ is a stopping time, $\theta_{T}: \Omega \rightarrow \Omega$ is defined by

$$
\left(\theta_{T} \omega\right) \equiv \theta_{T(\omega)}(\omega) .
$$

It is easy to verify the following result (cf. P. Courrège and P. Priouret [5] and E. B. Dynkin [7]). 
Proposition 2.6.1. (i) $\theta_{t}:\left(\Omega, \mathscr{F}_{t+s}\right) \rightarrow\left(\Omega, \mathscr{F}_{s}\right)$ is measurable, that is, $\theta_{t}^{-1} \mathscr{F}_{s} \subset \mathscr{F}_{t+s}$.

(ii) $\theta_{t}:\left(\Omega, \mathscr{M}_{t+s}\right) \rightarrow\left(\Omega, \mathscr{M}_{s}\right)$ is measurable, that is, $\theta_{t}^{-1} \mathscr{M}_{s} \subset \mathscr{M}_{t+s}$.

(iii) If $T$ is a stopping time, $\theta_{T}:\left(\Omega, \mathscr{M}_{T+s}\right) \rightarrow\left(\Omega, \mathscr{F}_{s}\right)$ is measurable, that is, $\theta_{T}^{-1} \mathscr{F}_{s} \subset \mathscr{M}_{T+s}$. Also $\theta_{T}^{-1} \mathscr{M}_{s} \subset \mathscr{M}_{T+s}$.

2.7. The zero-one law. The following zero-one law is well known.

Proposition 2.7.1. If $\left(\Omega, \mathscr{F}_{\infty},\left(P_{x}\right)_{x \in E_{\Delta}},\left(X_{t}\right)_{t \in[0, \infty]}\right)$ is a strong Markov process with respect to the $\sigma$-fields $\left(\mathscr{M}_{t}\right)_{t \in[0, \infty]}$ and $A \in \mathscr{M}_{0}$, then for each $x \in E_{\Delta}, P_{x}(A)$ is either zero or one.

Proof. Refer to E. B. Dynkin [7, Theorem 3.1]. It should also be noted that two zero-one measures on a $\sigma$-field which are equivalent are in fact identical.

2.8. The completed $\sigma$-fields. For any finite measure $\mu$ on $\left(E_{\Delta}, \mathscr{E}_{s}\right), P_{\mu}$ is a measure on $\left(\Omega, \mathscr{F}_{\infty}\right)$ defined by $P_{\mu}(A)=\int P_{x}(A) \mu(d x) . \mathscr{F}_{\infty}^{\pi}$ is defined to be the intersection of all the $P_{\mu}$-completions of $\mathscr{F}_{\infty}$. Each measure $P_{\mu}$ can be extended to $\mathscr{F}_{\infty}^{\pi}$. If $\mathscr{G}$ is a sub- $\sigma$-field of $\mathscr{F}_{\infty}, \tilde{\mathscr{G}}^{\pi}$ is defined to be such that $A \in \tilde{\mathscr{G}}^{\pi}$ if for each $\mu$ there exists a set $A_{\mu} \in \mathscr{G}$ such that $A-A_{\mu}$ and $A_{\mu}-A$ are in $\mathscr{F}_{\infty}^{\pi}$ and $P_{\mu}\left(A-A_{\mu}\right)=P_{\mu}\left(A_{\mu}-A\right)=0$. It can be shown that $\pi$ is also a strong Markov process with respect to the $\sigma$-fields $\left(\tilde{\mathscr{M}_{t}^{\pi}}\right)_{t \in[0, \infty]}$ (E. B. Dynkin [7, Theorem 3.12]). In the literature it is customary to replace the $\sigma$-fields $\left(\mathscr{M}_{t}\right)_{t \in[0, \infty]}$ by $\left(\tilde{\mathscr{M}}_{t}^{\pi}\right)_{t \in[0, \infty]}$. However, since if $\pi_{1}$ and $\pi_{2}$ are not equivalent $\tilde{\mathscr{M}}_{t}^{\pi_{1}}$ and $\tilde{\mathscr{M}}_{t}^{\pi}$ need not be identical, we avoid this convention unless it is explicitly stated to the contrary. However the following result is very useful.

Proposition 2.8.1. Let $T$ be an $\left(\tilde{\mathscr{M}}_{t}^{\pi}\right)_{t \in[0, \infty]}$-stopping time. Then for each $\mu$ there is $a\left(\mathscr{M}_{t}\right)_{t \in[0, \infty]}$-stopping time $T^{\mu}$ such that $P_{\mu}\left(T \neq T^{\mu}\right)=0$.

Proof. Refer to E. B. Dynkin [7, Lemma 3.4].

If $\Gamma$ is an $\mathscr{E}$-measurable set and

$$
\begin{aligned}
T_{\Gamma} & \equiv \inf \left\{t: X_{t}(\omega) \in \Gamma\right\} \text { if }\left\{t: X_{t}(\omega) \in \Gamma\right\} \neq \varnothing, \\
& \equiv+\infty \text { otherwise, }
\end{aligned}
$$

then $T_{\Gamma}$ is an $\left(\tilde{\mathscr{M}}_{t}^{\pi}\right)_{t \in[0, \infty}$-stopping time (P. A. Meyer [13, Chapter 4, T 52]. Note that if $\Gamma$ is compact, then $X_{T_{\Gamma}} \in \Gamma$ on $\left\{T_{\Gamma}<\infty\right\}$ and if $T_{\Gamma}^{\mu}$ is an $\left(\mathscr{M}_{t}\right)_{t \in[0, \infty}$-stopping time such that $P_{\mu}\left(T_{\Gamma} \neq T_{\Gamma}^{\mu}\right)=0$, then $X_{T_{\Gamma}^{\mu}} \in \Gamma, P_{\mu}$-almost surely on $\left\{T_{\Gamma}^{\mu}<\infty\right\}$.

2.9. Classification of stopping times. Let us briefly review P. A. Meyer's classification of stopping times [12], [13]. Let $\pi$ be a Hunt process. A stopping time $T$ is said to be totally inaccessible in the weak sense at $x_{0}$ on a set $A, x_{0} \in E, A \in \mathscr{F}_{\infty}$, if for every increasing sequence $\left\{T_{n}\right\}$ of stopping times which converge to $T$,

$$
P_{x_{0}}\left(\left\{T_{n}<T \text { for all } n, \quad T<\infty\right\} \cap A\right)=0 .
$$

A stopping time $T$ is said to be accessible at $x_{0}$ on a set $A, x_{0} \in E, A \in \mathscr{F}_{\infty}$, if there is an increasing sequence of stopping times $\left\{T_{n}\right\}$, with $T_{n} \leqq T$ for all $n$, and such that

$$
\lim _{n \rightarrow \infty} T_{n}=T
$$


$P_{x_{0}}$-almost surely on $A$, and

$$
T_{n}<T \quad \text { for all } n,
$$

$P_{x_{0}}$-almost surely on $A \cap\{T>0\}$. Note that every stopping time $T$ is accessible on

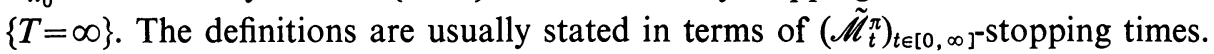
However in view of Proposition 2.8.1 this difference is inconsequential. The following result is of great importance.

Proposition 2.9.1. The stopping time $T$ is accessible at $x_{0}$ on $A$ if and only if $t \leadsto X_{t}$ is continuous at $T, P_{x_{0}}$-almost surely on $A \cap\{T<\infty\}$.

Proof. Refer to P. A. Meyer [12].

2.10. The definitions of equivalence. In the sequel $\pi_{1}=\left(\Omega, \mathscr{F}_{\infty},\left(P_{x}^{1}\right)_{x \in E_{\Delta}},\left(X_{t}\right)_{t \in[0, \infty]}\right)$ and $\pi_{2}=\left(\Omega, \mathscr{F}_{\infty},\left(P_{x}^{2}\right)_{x \in E_{\Delta}},\left(X_{t}\right)_{t \in[0, \infty]}\right)$ designate a pair of Hunt processes. $\pi_{1}$ and $\pi_{2}$ are said to be equivalent if for each $x \in E_{\Delta}$ and $A \in \mathscr{F}_{\infty}, P_{x}^{1}(A)=0$ if and only if $P_{x}^{2}(A)=0 . \pi_{1}$ and $\pi_{2}$ are equivalent in finite time if for each $x \in E_{\Delta}, t<\infty$ and $A \in \mathscr{M}_{t}$, $A \subset\{\zeta>t\}, P_{x}^{1}(A)=0$ if and only if $P_{x}^{2}(A)=0$; if this is true even if $A \nsubseteq\{\zeta>t\}, \pi_{1}$ and $\pi_{2}$ are strongly equivalent in finite time. A set $A \in \mathscr{F}_{\infty}$ such that $P_{x}^{1}(A)=0$, $P_{x}^{2}(A)>0$ is said to be a $\left(\pi_{1}, \pi_{2}, x\right)$-singularity.

\section{Three examples.}

EXAMPLE 1. Let $\pi=\left(\Omega, \mathscr{F}_{\infty},\left(P_{x}\right)_{x \in E_{\Delta}},\left(X_{t}\right)_{t \in[0, \infty]}\right)$ be the standard one-dimensional Brownian motion. Let $\pi_{1}=\left(\Omega, \mathscr{F}_{\infty},\left(P_{x}^{1}\right)_{x \in E_{\Delta}},\left(X_{t}\right)_{t \in[0, \infty]}\right)$ and $\pi_{2}=\left(\Omega, \mathscr{F}_{\infty},\left(P_{x}^{2}\right)_{x \in E_{\Delta}}\right.$, $\left.\left(X_{t}\right)_{t \in[0, \infty]}\right)$ be a pair of one-dimensional continuous strong Markov processes represented in the canonical form of 2.3 which are induced by the solutions of the stochastic integral equations:

$$
X_{1}(t)=X_{1}(0)+\int_{0}^{t} a_{1}\left(X_{1}(s)\right) d s+\int_{0}^{t} \sigma_{1}\left(X_{1}(s)\right) d X(s)
$$

and

$$
X_{2}(t)=X_{2}(0)+\int_{0}^{t} a_{2}\left(X_{2}(s)\right) d s+\int_{0}^{t} \sigma_{2}\left(X_{2}(s)\right) d X(s)
$$

where the stochastic integrals are evaluated with respect to the Brownian motion. In order to insure existence and uniqueness we also assume that

$$
a_{i}(\cdot) \text { and } \sigma_{i}(\cdot), \quad i=1,2 \text {, are continuous and } \sigma_{i}(\cdot)>0, \quad i=1,2,
$$

and

there exists a constant $K>0$ such that

$$
\left(a_{1}(x)\right)^{2}+\left(a_{2}(x)\right)^{2}+\left(\sigma_{1}(x)\right)^{2}+\left(\sigma_{2}(x)\right)^{2} \leqq K\left(1+x^{2}\right) .
$$

In the terminology of $\mathrm{K}$. Itô and H. P. McKean [11], the solutions $X_{1}(t)$ and $X_{2}(t)$ are nonstandard descriptions of $\pi_{1}$ and $\pi_{2}$.

A. V. Skorokhod [16], [17] has shown that $\pi_{1}$ and $\pi_{2}$ are strongly equivalent in 
finite time to the continuous strong Markov processes $\pi_{3}$ and $\pi_{4}$ which are induced by the unique solutions of the stochastic integral equations

$$
X_{3}(t)=X_{3}(0)+\int_{0}^{t} \sigma_{1}\left(X_{3}(s)\right) d X(s),
$$

and

$$
X_{4}(t)=X_{4}(0)+\int_{0}^{t} \sigma_{2}\left(X_{4}(s)\right) d X(s)
$$

respectively. A result originally due to V. A. Volkonskii (refer to K. Itô and H. P. McKean [11]) states that nonstandard descriptions of $\pi_{3}$ and $\pi_{4}$ are

$$
X_{i}\left(s_{i}\right)=X\left(f_{i}^{-1}\left(s_{i}\right)\right), \quad i=3,4,
$$

where $s_{i}=f_{i}(t, \omega), i=3,4$, are the additive functionals defined by

$$
f_{3}(t, \omega)=\int_{0}^{t} \sigma_{1}^{-2}(X(s, \sigma)) d s \text { and } f_{4}(t, \omega)=\int_{0}^{t} \sigma_{2}^{-2}(X(s, \omega)) d s
$$

where $X(s)$ represents the standard Brownian motion. In particular this implies that for any real number $a$

$$
P_{x_{0}}^{i}\left(\limsup _{t \downarrow 0} \frac{X(t)-x_{0}}{(2 t \log \log 1 / t)^{1 / 2}}=a\right)=1
$$

if and only if

$$
P_{x_{0}}\left(\limsup _{t \downarrow 0} \frac{X\left(f_{i}^{-1}(t)\right)-x_{0}}{(2 t \log \log 1 / t)^{1 / 2}}=a\right)=1, \quad i=3,4 .
$$

Proposition 3.1.

$$
P_{x_{0}}^{i}\left(\limsup _{t \downarrow 0} \frac{X(t)-x_{0}}{(2 t \log \log 1 / t)^{1 / 2}}=\sigma_{i}\left(x_{0}\right)\right)=1, \quad i=3,4 .
$$

Proof. Recall that the classical law of the iterated logarithm for Brownian motion states that

$$
P_{x_{0}}\left(\limsup _{s \downarrow 0} \frac{X(s)-x_{0}}{(2 s \log \log 1 / s)^{1 / 2}}=1\right)=1 .
$$

Hence it suffices to show that if $\omega_{0}$ is such that

$$
\limsup _{s \downarrow 0} \frac{X\left(s, \omega_{0}\right)-x_{0}}{(2 s \log \log 1 / s)^{1 / 2}}=1,
$$

then

$$
\limsup _{t \downarrow 0} \frac{X\left(f_{i}^{-1}\left(t, \omega_{0}\right)\right)-x_{0}}{(2 t \log \log 1 / t)^{1 / 2}}=\sigma_{i}\left(x_{0}\right), \quad i=3,4 .
$$

Because of the right continuity of paths, (3.8) is equivalent to

$$
\limsup _{t \downarrow 0} \frac{X\left(t, \omega_{0}\right)-x_{0}}{\left(2 s_{i}\left(t, \omega_{0}\right) \log \log 1 / s_{i}\left(t, \omega_{0}\right)\right)^{1 / 2}}=\sigma_{i}\left(x_{0}\right), \quad i=3,4 .
$$


Hence we must show that for $1>\varepsilon>0$,

$$
X\left(t, \omega_{0}\right)-x_{0}>\sigma_{i}\left(x_{0}\right)(1-\varepsilon)\left(2 s_{i}\left(t, \omega_{0}\right) \log \log 1 / s_{i}\left(t, \omega_{0}\right)\right)^{1 / 2}
$$

for arbitrarily small $t$, and

$$
X\left(t, \omega_{0}\right)-x_{0}<\sigma_{i}\left(x_{0}\right)(1+\varepsilon)\left(2 s_{i}\left(t, \omega_{0}\right) \log \log 1 / s_{i}\left(t, \omega_{0}\right)\right)^{1 / 2}
$$

for all sufficiently small $t$. We prove (3.10), (3.11) is proved in an analogous manner. Because of (3.7) we can assume that for arbitrarily small values of $t$,

$$
X\left(t, \omega_{0}\right)-x_{0}>(1-\varepsilon / 2)(2 t \log \log 1 / t)^{1 / 2} .
$$

Hence it suffices to show that for any $\rho$ satisfying $1>\rho>0$,

$$
\lim _{t \downarrow 0} \frac{(2 t \log \log 1 / t)^{1 / 2}}{\left(2 s_{i}\left(t, \omega_{0}\right) \log \log 1 / s_{t}\left(t, \omega_{0}\right)\right)^{1 / 2}} \geqq \sigma_{i}\left(x_{0}\right)(1-\rho), \quad i=3,4 .
$$

For $0<\eta_{i}<1 / \sigma_{i}^{2}\left(x_{0}\right)$ let $t_{0}$ be chosen so that for $t \leqq t_{0}$,

$$
\left|\sigma_{i}^{-2}\left(X\left(s_{i}\left(t, \omega_{0}\right)\right)\right)-\sigma_{i}^{-2}\left(x_{0}\right)\right|<\eta_{i}, \quad i=3,4 .
$$

But then if $t \leqq t_{0}, 0<t / \sigma_{i}^{2}\left(x_{0}\right)-\eta_{i} t \leqq s_{i}\left(t, \omega_{0}\right) \leqq t / \sigma_{i}^{2}\left(x_{0}\right)+\eta_{i} t$. But then

$$
\begin{aligned}
\left.\lim _{t \downarrow 0} \frac{(2 t}{\left(2 s_{i}\left(t, \omega_{0}\right)\right.} \log \log 1 / t\right)^{1 / 2} & \left.\log 1 / s_{i}\left(t, \omega_{0}\right)\right)^{1 / 2} \\
& \geqq \lim \frac{(2 t \log \log 1 / t)^{1 / 2}}{\left(2\left(t / \sigma_{i}^{2}\left(x_{0}\right)+\eta_{i} t\right) \log \log \left(1 /\left(t / \sigma_{i}^{2}\left(x_{0}\right)-\eta_{i} t\right)\right)\right)^{1 / 2}} \\
& =\frac{\sigma_{i}\left(x_{0}\right)}{\left(1+\eta_{i} \sigma_{i}^{2}\left(x_{0}\right)\right)^{1 / 2}} \lim _{t \downarrow 0}\left(\frac{\log \log 1 / t}{\log \log 1 / t\left(1 / \sigma_{i}^{2}\left(x_{0}\right)-\eta_{i}\right)}\right)^{1 / 2} \\
& =\frac{\sigma_{i}\left(x_{0}\right)}{\left(1+\eta_{i} \sigma_{i}^{2}\left(x_{0}\right)\right)^{1 / 2}}, \quad i=3,4, \\
& \geqq(1-\rho) \sigma_{i}\left(x_{0}\right) \quad \text { if } \eta_{i},(i=3,4), \text { are chosen to be sufficiently small. }
\end{aligned}
$$

The last limit indicated above is found by a double application of De l'Hôpital's rule. Hence (3.10) follows and the proof is complete.

COROLlaRY. $\pi_{1}$ and $\pi_{2}$ are equivalent in finite time if and only if they are instantaneously equivalent.

Proof. Since

$$
\left\{\limsup _{t \downarrow 0} \frac{X(t)-X(0)}{(2 t \log \log 1 / t)^{1 / 2}}=a\right\} \in \mathscr{M}_{0}
$$

the proposition implies that if $\pi_{1}$ and $\pi_{2}$ are instantaneously equivalent, then $\sigma_{1}(x)=\sigma_{2}(x)$ for all $x$. On the other hand if $\sigma_{1}(x)=\sigma_{2}(x)$ for all $x \in E$, then the results of A. V. Skorokhod imply that $\pi_{1}$ and $\pi_{2}$ are strongly equivalent in finite time.

REMARKS. 3.1. It can be shown that if $a_{1}(x) \geqq a_{2}(x)+c$ for all $x \in E$ where $c$ is a 
constant, then $\pi_{1}$ and $\pi_{2}$ are not equivalent on $\mathscr{F}_{\infty}$ even when $\sigma_{1}(x)=\sigma_{2}(x)$ for all $x \in E$.

3.2. In the above proposition it is shown that for a one-dimensional diffusion the measures restricted to the germ fields actually determine the diffusive part of the infinitesimal generator and thus determine the equivalence class (in finite time) of the process. However the extension of this result to higher dimensions has not yet been established. Nevertheless one would expect the extension to be valid and our results on equivalence lend support to this supposition.

The following two examples show that instantaneous equivalence does not always imply equivalence in finite time and illustrate two of the types of singularities which are discussed in $\S 8$.

EXAMPLE 2. An example of a pair of strong Markov processes which are instantaneously equivalent but not equivalent in finite time is furnished by the pair of deterministic processes:

$$
X_{1}(t) \equiv X_{1}(0)+t
$$

and

$$
X_{2}(t) \equiv X_{2}(0)+t-n\left(t+X_{2}(0)\right)+n\left(X_{2}(0)\right),
$$

where $n(t)$ designates the greatest integer less than or equal to $t$. Note that the second process defined by (3.14) is not quasi-left continuous.

EXAMPLE 3. Let $\pi=\left(\Omega, \mathscr{F}_{\infty},\left(P_{x}\right)_{x \in E_{\Delta}},\left(X_{t}\right)_{t \in[0, \infty]}\right)$ be a Hunt process such that for some $x_{0} \in E$ and $t_{0}<\infty, P_{x_{0}}\left(\zeta<t_{0}\right)>0$. Let $E_{\Delta, \Delta^{\prime}}=E \cup\{\Delta\} \cup\left\{\Delta^{\prime}\right\}$ where $\Delta^{\prime}$ is an isolated point and $\Delta^{\prime} \notin E \cup\{\Delta\}$. Let $\Omega^{\prime}$ be the set of right continuous functions, $\omega(\cdot)$, from $[0, \infty]$ to $E_{\Delta, \Delta^{\prime}}$ such that:

$$
\begin{aligned}
& \omega(\cdot) \text { has left-hand limits on }[0, \infty) \text {, } \\
& \text { if } \omega(t)=\Delta \text {, then } \omega(s)=\Delta \quad \text { for all } s \geqq t \text {, } \\
& \text { if } \omega(t)=\Delta^{\prime} \text {, then } \omega(s)=\Delta^{\prime} \quad \text { for all } s \geqq t \text {, and } \\
& \omega(\infty) \in\{\Delta\} \cup\left\{\Delta^{\prime}\right\} .
\end{aligned}
$$

Let $\mathscr{F}_{\infty}^{\prime}$ be the $\sigma$-field generated by the random variables $\left(X_{t}\right)_{t \in[0, \infty]}$. The measures $\left(P_{x}\right)_{x \in E}$ can be extended to $\left(\Omega^{\prime}, \mathscr{F}_{\infty}^{\prime}\right)$ by defining $P_{x}^{1}(A) \equiv P_{x}(A \cap \Omega)$ for $A \in \mathscr{F}_{\infty}^{\prime}$. Let $\pi_{1} \equiv\left(\Omega^{\prime}, \mathscr{F}_{\infty}^{\prime},\left(P_{x}^{1}\right)_{x \in E_{\Delta, \Delta}},\left(X_{t}\right)_{t \in[0, \infty]}\right)$. Let $\Phi: \Omega^{\prime} \rightarrow \Omega^{\prime}$ be defined by:

and

$$
\begin{array}{ll}
\Phi(\omega)(s)=\omega(s) & \text { if } \omega(s) \in E, \\
\Phi(\omega)(s)=\Delta^{\prime} & \text { if } \omega(s)=\Delta,
\end{array}
$$

$$
\Phi(\omega)(s)=\Delta \quad \text { if } \omega(s)=\Delta^{\prime} .
$$

For $A \in \mathscr{F}_{\infty}^{\prime}$, let $P_{x}^{2}(A) \equiv P_{x}^{1}\left(\Phi^{-1}(A)\right)$ and let $\pi_{2}=\left(\Omega^{\prime}, \mathscr{F}_{\infty}^{\prime},\left(P_{x}^{2}\right)_{x \in E_{\Delta, \Delta}},\left(X_{t}\right)_{t \in[0, \infty]}\right)$. Then $\pi_{1}$ and $\pi_{2}$ are instantaneously equivalent and even equivalent in finite time. However they are not strongly equivalent in finite time, in fact $P_{x_{0}}^{1}\left(X_{t_{0}}=\Delta\right)>0$, $P_{x_{0}}^{2}\left(X_{t_{0}}=\Delta\right)=0$. 
4. The stochastic processes $Y_{A}(t, \cdot)$. In this section we assign to each Hunt process $\pi=\left(\Omega, \mathscr{F}_{\infty},\left(P_{x}\right)_{x \in E_{\Delta}},\left(X_{t}\right)_{t \in[0, \infty]}\right)$ and set $A \in \mathscr{F}_{\infty}$ a stochastic process $Y_{A}(t, \cdot)$. These processes are introduced in order to formulate an extended Markov property in $\S 5$. The extended Markov property implies that $Y_{A}(t, \cdot)$ is for each $x \in E$, a version of the martingale $P_{x}\left(A \mid \mathscr{M}_{t}\right)$.

Before introducing the processes $Y_{A}(t, \cdot)$ it is necessary to review some useful notation and results due to P. Courrège and P. Priouret [5]. If $H: \Omega \rightarrow[0, \infty]$ is measurable, an equivalence relation $R_{H}$ on $\Omega$ is defined by:

$$
\begin{aligned}
& \omega \sim \omega^{\prime}\left(R_{H}\right) \text { if and only if } H(\omega)=H\left(\omega^{\prime}\right) \text { and } \\
& X_{s}(\omega)=X_{s}\left(\omega^{\prime}\right) \text { for all } s \leqq H(\omega) .
\end{aligned}
$$

A second equivalence relation $R_{H+}$ is defined by:

$\omega \sim \omega^{\prime}\left(R_{H+}\right)$ if and only if $H(\omega)=H\left(\omega^{\prime}\right)$ and there exists an $\varepsilon>0$ such that $X_{s}(\omega)=X_{s}\left(\omega^{\prime}\right)$ for all $s \leqq H(\omega)+\varepsilon$.

Proposition 4.1. (i) $\mathscr{F}_{t}$ is the sub- $\sigma$-field of sets of $\mathscr{F}_{\infty}$ which are saturated for $R_{t}$.

(ii) $\mathscr{M}_{t}$ is the sub- $\sigma$-field of sets of $\mathscr{F}_{\infty}$ saturated for $R_{t+}$.

(iii) If $T$ is an $\left(\mathscr{M}_{t}\right)$-stopping time, then $\mathscr{M}_{T}$ is the sub- $\sigma$-field of sets of $\mathscr{F}_{\infty}$ saturated for $R_{T+}$.

The following version of Galmarino's lemma will also be required.

Proposition 4.2. In order that $T: \Omega \rightarrow[0, \infty]$ be an $\left(\mathscr{M}_{t}\right)$-stopping time it is necessary and sufficient that $T$ be $\mathscr{F}_{\infty}$-measurable and that for each $t \in[0, \infty]$, $\omega \in \Omega, \omega^{\prime} \in \Omega, T(\omega)<t$ and $\omega^{\prime} \sim \omega\left(R_{t}\right)$ implies that $T\left(\omega^{\prime}\right)=T(\omega)$.

Proof. Refer to P. Courrège and P. Priouret [5].

Let $A \in \mathscr{F}_{\infty}$ and $\omega \in \Omega$. Then for $t \in[0, \infty)$,

$$
A^{*}(t, \omega) \equiv\left\{\omega^{\prime}: \omega^{\prime} \in A, \omega^{\prime} \sim \omega\left(R_{t}\right)\right\}
$$

and

$$
A(t, \omega) \equiv \theta_{t} A^{*}(t, \omega)=\left\{\theta_{t} \omega^{\prime}: \omega^{\prime} \in A^{*}(t, \omega)\right\}
$$

and for $t=\infty$,

$$
A(\infty, \omega) \equiv \varnothing \quad \text { if } \omega \notin A,
$$

and

$$
A(\infty, \omega) \equiv\left\{\omega_{\Delta}\right\} \quad \text { if } \omega \in A .
$$

The fact that $A(t, \omega) \in \mathscr{F}_{\infty}$ is verified in the following proposition.

Proposition 4.3. (i) If $A \in \mathscr{F}_{s}$ and $t<s$, then for each $\omega \in \Omega, A(t, \omega) \in \mathscr{F}_{s-t}$.

(ii) $\left(\bigcup_{i=1}^{\infty} A_{i}\right)(t, \omega)=\bigcup_{i=1}^{\infty} A_{i}(t, \omega)$ for each $\omega \in \Omega$.

(iii) $\left(\bigcap_{i=1}^{\infty} A_{i}\right)(t, \omega)=\bigcap_{i=1}^{\infty} A_{i}(t, \omega)$ for each $\omega \in \Omega$.

(iv) $A^{C}(t, \omega)=(A(t, \omega))^{C} \cap\left\{\omega^{\prime}: X_{0}\left(\omega^{\prime}\right)=X_{t}(\omega)\right\}$ for each $\omega \in \Omega$. 
Proof.

(ii) $\left(\bigcup_{i=1}^{\infty} A_{i}\right)(t, \omega)=\theta_{t}\left[\left(\bigcup_{i=1}^{\infty} A_{i}\right)^{*}(t, \omega)\right]=\theta_{t}\left[\bigcup_{i=1}^{\infty} A_{i}^{*}(t, \omega)\right]=\bigcup_{i=1}^{\infty} \theta_{t} A_{i}^{*}(t, \omega)$ $=\bigcup_{i=1}^{\infty} A_{i}(t, \omega)$.

(iii) $\left(\bigcap_{i=1}^{\infty} A_{i}\right)(t, \omega)=\theta_{t}\left[\left(\bigcap_{i=1}^{\infty} A_{i}\right)^{*}(t, \omega)\right]=\theta_{t}\left[\bigcap_{i=1}^{\infty} A_{i}^{*}(t, \omega)\right]=\bigcap_{i=1}^{\infty} \theta_{t} A_{i}^{*}(t, \omega)$ $=\bigcap_{i=1}^{\infty} A_{i}(t, \omega)$.

(iv) $A^{C}(t, \omega)=\theta_{t}\left(A^{C^{*}}(t, \omega)\right)=\theta_{t}\left[\left(A^{*}(t, \omega)\right)^{C} \cap\left\{\omega^{\prime}: X_{t}\left(\omega^{\prime}\right)=X_{t}(\omega)\right\}\right]=(A(t, \omega))^{C} \cap$ $\left\{\omega^{\prime}: X_{0}\left(\omega^{\prime}\right)=X_{t}(\omega)\right\}$.

To prove (i) it thus suffices to show that $A(t, \omega) \in \mathscr{F}_{s-t}$ when

$$
A=\left\{\omega: X_{v}(\omega) \in \Gamma_{1}, X_{t}(\omega) \in \Gamma_{2}, X_{u}(\omega) \in \Gamma_{3}\right\}
$$

where $\Gamma_{1}, \Gamma_{2}, \Gamma_{3}$ are in $\mathscr{E}$ and $v<t<u \leqq s$. But then

$$
\begin{aligned}
A(t, \omega) & =\left\{\omega^{\prime}: X_{0}\left(\omega^{\prime}\right)=X_{t}(\omega), X_{u-t}\left(\omega^{\prime}\right) \in \Gamma_{3}\right\} \\
& =\varnothing \quad \text { if } X_{v}(\omega) \notin \Gamma_{1} \text { or } X_{t}(\omega) \notin \Gamma_{2} .
\end{aligned}
$$

Hence $A(t, \omega) \in \mathscr{F}_{s-t}$ and the proof is complete.

For $A \in \mathscr{F}_{\infty}$ and Hunt process $\pi=\left(\Omega, \mathscr{F}_{\infty},\left(P_{x}\right)_{x \in E_{\Delta}},\left(X_{t}\right)_{t \in[0, \infty]}\right)$ the real-valued stochastic process $\left(\Omega, \mathscr{F}_{\infty},\left(P_{s}\right)_{x \in E_{\Delta}},\left(Y_{A}(t)\right)_{t \in[0, \infty]}\right)$ is defined as follows:

$$
Y_{A}(t, \omega) \equiv P_{X(t, \omega)}(A(t, \omega)) \quad \text { for } t \in[0, \infty], \omega \in \Omega \text {. }
$$

Note that $Y_{\Omega}(t, \omega) \equiv 1$.

Proposition 4.4. (i) If $A \cap B=\varnothing$, then $Y_{A \cup B}(t, \omega)=Y_{A}(t, \omega)+Y_{B}(t, \omega)$ for each $\omega \in \Omega$ and $t \in[0, \infty]$.

(ii) If $A_{i} \uparrow A$ or $A_{i} \downarrow A$, then $Y_{A}(t, \omega)=\lim _{i \rightarrow \infty} Y_{A_{i}}(t, \omega)$ for each $\omega \in \Omega$ and $t \in[0, \infty]$.

(iii) If $A \in \mathscr{F}_{\infty}$, then $Y_{A}(t, \cdot)$ is measurable with respect to $\mathscr{F}_{t}$.

Proof. (i) By Proposition 4.3, (ii) and (iv), if $A \cap B=\varnothing$, then

$$
\begin{aligned}
Y_{A \cup B}(t, \omega) & =P_{X(t, \omega)}((A \cup B)(t, \omega)) \\
& =P_{X(t, \omega)}(A(t, \omega))+P_{X(t, \omega)}(B(t, \omega))=Y_{A}(t, \omega)+Y_{B}(t, \omega) .
\end{aligned}
$$

(ii) We prove (ii) for $A_{i} \uparrow A$, the proof for $A_{i} \downarrow A$ is similar. If $A_{i} \uparrow A$, then by Proposition 4.3. (i), $A_{i}(t, \omega) \uparrow A(t, \omega)$. But then

$$
Y_{A_{i}}(t, \omega)=P_{X(t, \omega)}\left(A_{i}(t, \omega)\right) \uparrow P_{X(t, \omega)}(A(t, \omega))=Y_{A}(t, \omega) .
$$

In view of (i) and (ii) it suffices to prove (iii) for a set of the form

$$
A=\left\{X\left(t_{1}\right) \in \Gamma_{1}, \ldots, X\left(t_{i}\right) \in \Gamma_{i}, X(t) \in \Gamma_{0}, X\left(t_{i+1}\right) \in \Gamma_{i+1}, \ldots, X\left(t_{n}\right) \in \Gamma_{n}\right\}
$$

where $\Gamma_{i} \in \mathscr{E}, i=0, \ldots, n$ and $t_{1}<t_{2}<\cdots<t_{i}<t<t_{i+1}<\cdots<t_{n}$. But then

$$
\begin{aligned}
& A(t, \omega)=\left\{X\left(t_{i+1}-t\right) \in \Gamma_{i+1}, \ldots, X\left(t_{n}-t\right) \in \Gamma_{n}\right\} \cap\left\{X(0)=X_{t}(\omega)\right\} \\
& \text { if } \omega \in\left\{X\left(t_{1}\right) \in \Gamma_{1}, \ldots, X\left(t_{i}\right) \in \Gamma_{i}, X(t) \in \Gamma_{0}\right\}, \\
&=\varnothing \quad \text { if } \omega \notin\left\{X\left(t_{1}\right) \in \Gamma_{1}, \ldots, X\left(t_{i}\right) \in \Gamma_{i}, X(t) \in \Gamma_{0}\right\} .
\end{aligned}
$$


Hence

$$
\begin{aligned}
P_{X(t, \omega)}(A(t, \omega))= & \chi_{\left(X\left(t_{1}\right) \in \Gamma_{1}, \ldots, X\left(t_{i}\right) \in \Gamma_{i}, X(t) \in \Gamma_{0}\right\}} \\
& \cdot P_{X(t, \omega)}\left(X\left(t_{i+1}-t\right) \in \Gamma_{i+1}, \ldots, X\left(t_{n}-t\right) \in \Gamma_{n}\right),
\end{aligned}
$$

where $\chi_{K}(\cdot)$ designates the indicator function of the set $K$. The result then follows since

$$
P_{X(t, \omega)}\left(X\left(t_{i+1}-t\right) \in \Gamma_{i+1}, \ldots, X\left(t_{n}-t\right) \in \Gamma_{n}\right)
$$

is a composition of the $\mathscr{F}_{t}$-measurable mapping $\omega \leadsto X_{t}(\omega)$ and the measurable mapping $y \leadsto P_{y}\left(X\left(t_{i+1}-t\right) \in \Gamma_{i+1}, \ldots, X\left(t_{n}-t\right) \in \Gamma_{n}\right)$.

Proposition 4.5. If $A \in \mathscr{F}_{\infty}$, then $Y_{A}(\cdot, \cdot)$ is progressively measurable.

Proof. Proposition 4.4 implies that the class of sets $A$, for which $Y_{A}(\cdot, \cdot)$ is progressively measurable is closed under the operations of taking complements, disjoint unions and monotone limits. Since $E$ is a locally compact separable metric space, it suffices to show that $Y_{A}(\cdot, \cdot)$ is progressively measurable for a set of the form

$$
A=\left\{X\left(t_{1}\right) \in \Gamma_{1}, \ldots, X\left(t_{m}\right) \in \Gamma_{m}\right\}
$$

with $\Gamma_{1}, \ldots, \Gamma_{m}$ compact and $t_{1}<t_{2}<\cdots<t_{m}$. For $t_{i-1} \leqq s<t$

$$
Y_{A}(s, \omega)=\chi_{\left\{X\left(t_{1}\right) \in \Gamma_{1}\right\}} \cdots \chi_{\left(X\left(t_{i-1}\right) \in \Gamma_{i-1}\right)} \cdot P_{X(s, \omega)}\left(X\left(t_{i}-s\right) \in \Gamma_{i}, \ldots, X\left(t_{m}-s\right) \in \Gamma_{m}\right) .
$$

Since the $\Gamma_{i}, i=1, \ldots, n$ are compact, for each $i$ there is a sequence $\left\{f_{i}^{n}\right\}$ of $C_{0}(E)$ functions such that $f_{i}^{n}(x) \downarrow \chi_{\Gamma_{i}}(x)$ as $n \rightarrow \infty$ (P. R. Halmos [9, Chapter 10]). But then

$$
\begin{aligned}
P_{X(s, \omega)}\left(X\left(t_{i}-s\right) \in \Gamma_{i}, \ldots\right. & \left., X\left(t_{m}-s\right) \in \Gamma_{m}\right) \\
& =\lim _{n \rightarrow \infty} E_{X(s, \omega)}\left(f_{i}^{n}\left(X\left(t_{i}-s\right)\right) \cdot f_{i+1}^{n}\left(X\left(t_{i+1}-s\right)\right) \cdots f_{m}^{n}\left(X\left(t_{m}-s\right)\right)\right) \\
& =\lim _{n \rightarrow \infty} P_{t_{i}-s}\left(f_{i}^{n}\left(y_{i}\right) \cdots\left(P_{t_{m}-t_{m-1}} f_{m}^{n}\left(y_{m}\right)\right)\right)(X(s, \omega)) .
\end{aligned}
$$

But Proposition 2.4.1 implies that $P_{t_{i}-s}\left(f_{i}^{n}\left(y_{i}\right) \cdots\left(P_{t_{m}-t_{m-1}} f_{m}^{n}\left(y_{m}\right)\right)\right)(y)$ is jointly continuous with respect to $s$ and $y$ for $s \in\left[t_{t-1}, t_{i}\right)$ and $y \in E$. Since $X(s, \omega)$ is a right continuous function of $s$ for each $\omega, P_{t_{i}-s}\left(f_{i}^{n}\left(y_{i}\right) \cdots\left(P_{t_{m}-t_{m-1}} f_{m}^{n}\left(y_{m}\right)\right)\right)(X(s \omega))$ is therefore right continuous for $s \in\left[t_{i-1}, t_{i}\right)$ for each $\omega$. Hence $Y_{A}(\cdot, \cdot)$ is progressively measurable (cf. P. A. Meyer [13, Chapter 4, T 47]).

We now introduce the concept of a relative stopping time. Let $T$ be a stopping time and let $t \leqq T\left(\omega_{0}\right), \omega_{0} \in \Omega$. Note that if $\omega \in \Omega$ and $X_{0}(\omega)=X_{t}\left(\omega_{0}\right)$ then there is a path $\omega^{\prime} \in \Omega$ such that $\omega^{\prime} \sim \omega_{0}\left(R_{t}\right)$ and such that $\omega=\theta_{t} \omega^{\prime}$. In this case define $T^{\prime}(\omega) \equiv T\left(\omega^{\prime}\right)-t$. If $X_{0}(\omega) \neq X_{t}\left(\omega_{0}\right)$ let $T^{\prime}(\omega) \equiv+\infty$. $T^{\prime}$ is called the $\left(T, \omega_{0}, t\right)-$ relative stopping time.

Proposition 4.6. The $\left(T, \omega_{0}, t\right)$-relative stopping time is a stopping time. 
Proof. It is easy to verify that $T^{\prime}$ is a measurable mapping from $\Omega$ to $[0, \infty]$. In particular, for $s<\infty,\left\{T^{\prime}<s\right\}=\left\{X(0)=X\left(t, \omega_{0}\right)\right\} \cap\{T<s+t\}(t, \omega)$. The result then follows from Proposition 4.3 which implies that $\{T<s+t\}(t, \omega) \in \mathscr{M}_{s}$.

5. The extended Markov property. In this section the extended Markov property is established. However, before doing this it is necessary to introduce a new class of $\sigma$-fields.

Given a stopping time $T, \mathscr{M}_{T}^{*}$ is the $\sigma$-field $\theta_{T}^{-1} \mathscr{F}_{\infty}$ which is a sub- $\sigma$-field of $\mathscr{F}_{\infty}$ (Proposition 2.6.1). It is easy to verify that $\mathscr{M}_{T}^{*}$ is the $\sigma$-field generated by the random variables $\left(X_{T+s}\right)_{s \geqq 0}$.

Proposition 5.1. For any $\left(\mathscr{M}_{t}\right)$-stopping time $T, \mathscr{M}_{T} \vee \mathscr{M}_{T}^{*}$, the $\sigma$-field generated by $\mathscr{M}_{T}$ and $\mathscr{M}_{T}^{*}$ is identical to $\mathscr{F}_{\infty}$.

Proof. Clearly $\mathscr{M}_{T} \vee \mathscr{M}_{T}^{*} \subset \mathscr{F}_{\infty}$. Hence it suffices to show that $\left\{X_{t} \in \Gamma\right\}$ for $t \geqq 0$ and $\Gamma$ an open subset of $E$ is measurable with respect to $\mathscr{M}_{T} \vee \mathscr{M}_{T}^{*}$.

For $\Gamma \in \mathscr{E}$,

$$
\left\{X_{t} \in \Gamma\right\}=\left(\{T \geqq t\} \cap\left\{X_{t} \in \Gamma\right\}\right) \cup\left(\{T<t\} \cap\left\{X_{t} \in \Gamma\right\}\right) .
$$

But $\{T \geqq t\} \cap\left\{X_{t} \in \Gamma\right\} \in \mathscr{M}_{T}$. Hence it suffices to show that $\{T<t\} \cap\left\{X_{t} \in \Gamma\right\} \in \mathscr{M}_{T}^{*}$. Note that $T_{n} \equiv \sum_{i=0}^{\infty} \chi_{[i / n \leqq T<(i+1) / n]}((i+1) / n), n=1,2,3, \ldots$, is a decreasing sequence of stopping times with limit $T$. Then if $\eta_{n} \equiv\left(T+t-T_{n}+1 / n\right) \vee T, \eta_{n} \rightarrow t \vee T$, and the $\eta_{n}, n=1,2,3, \ldots$, are measurable with respect to $\mathscr{M}_{T}$. Hence the $\eta_{n}, n=1,2,3, \ldots$, are stopping times (P. A. Meyer [1, Chapter 4, T 38]). The right continuity of the paths and the fact that $\Gamma$ is open imply that

$$
\left\{X_{t} \in \Gamma\right\} \cap\{T<t\}=\bigcup_{j \in \mathscr{J}} \bigcup_{k=1}^{\infty} \bigcap_{n=k}^{\infty}\left(\{T<t\} \cap\left\{X_{n_{n}} \in \Gamma_{j}\right\}\right)
$$

where the $\Gamma_{j}, j=1,2,3, \ldots$, and $\mathscr{J}$ are chosen exactly as in the proof of Proposition 2.2.1. But for each $n$ and $j$,

$$
\begin{aligned}
\{T<t\} \cap\left\{X_{n_{n}} \in \Gamma_{j}\right\} & =\bigcup_{i=0}^{\infty}\left(\{T<t\} \cap\left\{X_{n_{n}} \in \Gamma_{j}\right\} \cap\left\{T_{n}=i / n\right\}\right) \\
& =\bigcup_{i=0}^{\infty}\left(\{T<t\} \cap\left\{X(T+t-(i-1) / n) \in \Gamma_{j}\right\} \cap\left\{T_{n}=1 / n\right\}\right) .
\end{aligned}
$$

Since $t \geqq(i-1) / n$ on $\{T<t\} \cap\left\{T_{n}=i / n\right\}$, therefore

$$
\{T<t\} \cap\left\{X(T+(t-(i-1) / n)) \in \Gamma_{j}\right\} \cap\left\{T_{n}=i / n\right\} \in \mathscr{M}_{T}^{*} .
$$

Hence $\{T<t\} \cap\left\{X_{t} \in \Gamma\right\} \in \mathscr{M}_{T}^{*}$ and the proof is complete. The following corollary has also been proved.

COROllaRY. For any stopping time $T$ the events of the form $\left\{X_{T+t} \in \Gamma\right\}$ with $\Gamma \in \mathscr{E}$ and $t \geqq 0$ and $\left\{X_{t} \in \Gamma\right\} \cap\{T \geqq t\}$ with $\Gamma \in \mathscr{E}$ and $t \geqq 0$ generate the $\sigma$-field $\mathscr{F}_{\infty}$. 
Theorem 5.1 (The Extended Markov Property). For any stopping time T, $A \in \mathscr{F}_{\infty}, B \in \mathscr{M}_{T}$ and any $x \in E$,

$$
\begin{aligned}
P_{x}(A \cap B) & =\int_{B} P_{X(T)}(A(T(\omega), \omega)) d P_{x}(\omega) \\
& =\int_{B} Y_{A}(T(\omega), \omega) d P_{x}(\omega) \\
& =E_{x}\left(\chi_{B}(\omega) \cdot Y_{A}(T(\omega), \omega)\right) .
\end{aligned}
$$

In other words for every $x \in E, Y_{A}(t, \cdot)$ is a version of the martingale $E_{x}\left(\chi_{A} \mid \mathscr{M}_{t}\right)$ for which the optional sampling theorem is valid.

Proof. $P_{x}(A \cap B)=P_{x}(A \cap B \cap\{T<\infty\})+P_{x}(A \cap B \cap\{T=\infty\})$. But

$$
\begin{aligned}
P_{x}(A \cap B \cap\{T=\infty\}) & =\int_{B \cap\{T=\infty\}} \chi_{A} \cdot P_{\Delta}\left(\omega_{\Delta}\right) d P_{x}(\omega) \\
& =E_{x}\left(\chi_{B \cap\{T=\infty\}} \cdot Y_{A}(T(\omega), \omega)\right) .
\end{aligned}
$$

Hence it suffices to prove (5.1) in the case in which $B \subset\{T<\infty\}$. Note that since $Y_{A}(\cdot, \cdot)$ is progressively measurable, $Y_{A}(T(\omega), \omega)$ is measurable with respect to $\mathscr{M}_{T}$ and hence the integral on the right-hand side of (5.1) is well defined.

We first show that the class of sets $A$ for which (5.1) is satisfied is closed under disjoint unions and monotone limits. If $A \cap C=\varnothing$ and both $A$ and $C$ satisfy (5.1), then

$$
\begin{aligned}
P_{x}(B \cap(A \cup C)) & =P_{x}(A \cap B)+P_{x}(C \cap B) \\
& =\int_{B} Y_{A}(T(\omega), \omega) d P_{x}(\omega)+\int_{B} Y_{C}(T(\omega), \omega) d P_{x}(\omega) \\
& =\int_{B} Y_{A \cup C}(T(\omega), \omega) d P_{x}(\omega) \quad \text { by Proposition 4.4, }
\end{aligned}
$$

and therefore $A \cup C$ satisfies (5.1). If $A_{i} \downarrow A$ as $i \rightarrow \infty$ and $A_{i}, i=1,2,3, \ldots$, satisfy (5.1), then

$$
\begin{aligned}
P_{x}(A \cap B) & =\lim _{i \rightarrow \infty} P_{x}\left(A_{i} \cap B\right)=\lim _{i \rightarrow \infty} \int_{B} Y_{A_{i}}(T(\omega), \omega) d P_{x}(\omega) \\
& =\int_{B} \lim _{i \rightarrow \infty} Y_{A_{t}}(T(\omega), \omega) d P_{x}(\omega) \quad \text { by the monotone convergence theorem } \\
& =\int_{B} Y_{A}(T(\omega), \omega) d P_{x}(\omega) \quad \text { by Proposition 4.4. }
\end{aligned}
$$

Therefore $A$ satisfies (5.1).

Hence in view of Proposition 5.1 it suffices to prove (5.1) for sets of the form

$$
A_{0}=C \cap\left\{X\left(T+t_{1}\right) \in \Gamma_{1}\right\} \cap \cdots \cap\left\{X\left(T+t_{n}\right) \in \Gamma_{n}\right\},
$$

where $0 \leqq t_{1}<t_{2} \ldots<t_{n}, \Gamma_{i} \in \mathscr{E}, i=1, \ldots, n$, and $C \in \mathscr{M}_{T}$. In fact in view of the Corollary to Proposition 5.1, it suffices to consider sets $C$ of the form

$$
C=\left\{X\left(s_{1}\right) \in \Lambda_{1}, \ldots, X\left(s_{m}\right) \in \Lambda_{m}\right\} \cap\left\{s_{m} \leqq T<s_{m+1}\right\},
$$


where $0 \leqq s_{1}<\cdots<s_{m+1}$ and $\Lambda_{1} \in \mathscr{E}, i=1, \ldots, m$. In this case it is easy to verify that the strong Markov property implies that

$$
P_{x}\left(A_{0} \cap B\right)=\int_{B \cap C} P_{X(T(\omega))}\left(X\left(t_{1}\right) \in \Gamma_{1}, \ldots, X\left(t_{n}\right) \in \Gamma_{n}\right) d P_{x}(\omega),
$$

(cf. E. B. Dynkin [7, Theorem 3.11]). Hence it suffices to show that if $A_{0}$ is defined by (5.2) then

$$
\int_{B \cap C} P_{X(T(\omega))}\left(X\left(t_{1}\right) \in \Gamma_{1}, \ldots, X\left(t_{n}\right) \in \Gamma_{n}\right) d P_{x}(\omega)=\int_{B} Y_{A_{0}}(T(\omega), \omega) d P_{x}(\omega) .
$$

Let $H=\left\{X_{T}^{-}=X_{T}\right\} \cap\{T<\infty\}$ and $F \equiv\left\{X_{T}^{-} \neq X_{T}\right\} \cap\{T<\infty\}$. For $k=1,2,3, \ldots$, define

$$
\begin{aligned}
J_{0}^{k}(\cdot) & =0 \\
J_{n+1}^{k}(\omega) & =\inf \left\{s: s>J_{n}^{k}(\omega), \rho\left(X_{s}(\omega), X_{s}^{-}(\omega)\right)>1 / k\right\} \\
& =+\infty \quad \text { if the above set is empty. }
\end{aligned}
$$

Since the paths possess limits from the left on $[0, \infty)$ and are right continuous,

$$
F=\left[\left(\bigcup_{k=1}^{\infty} \bigcup_{n=0}^{\infty}\left\{T=J_{n}^{k}\right\}\right) \cup(F \cap\{T=\zeta\})\right] \cap\{T<\infty\} .
$$

Note that $\left\{T=J_{n}^{k}\right\} \in \mathscr{M}_{J_{n}^{k}} \cap \mathscr{M}_{T}$ for any $k$ and $n$. If $\omega \in \Omega$ and if $\omega \sim \omega^{\prime}\left(R_{J_{n}^{k}}\right)$, then $J_{n}^{k}\left(\omega^{\prime}\right)=J_{n}^{k}(\omega)$.

We now prove (5.1) for the case $T \equiv J_{n}^{k}$ for some $k$ and $n$. If $A_{0}$ is defined by (5.2) (with $T \equiv J_{n}^{k}$ ), then

$$
\begin{aligned}
A_{0}\left(J_{n}^{k}(\omega), \omega\right) & =\left\{X\left(t_{1}\right) \in \Gamma_{1}\right\} \cap \cdots \cap\left\{X\left(t_{n}\right) \in \Gamma_{n}\right\} \cap\left\{X(0)=X\left(J_{n}^{k}(\omega)\right)\right\} \quad \text { if } \omega \in C \\
& =\varnothing \quad \text { if } \omega \notin C,
\end{aligned}
$$

and therefore

$$
Y_{A_{0}}\left(J_{n}^{k}(\omega), \omega\right)=\chi_{C} \cdot P_{X\left(J_{n}^{k}(\omega)\right)}\left(X\left(t_{1}\right) \in \Gamma_{1}, \ldots, X\left(t_{n}\right) \in \Gamma_{n}\right) .
$$

Therefore

$$
\int_{B \cap C} P_{X\left(J_{n}^{k}(\omega)\right)}\left(X\left(t_{1}\right) \in \Gamma_{1}, \ldots, X\left(t_{n}\right) \in \Gamma_{n}\right) d P_{x}(\omega)=\int_{B} Y_{A_{0}}\left(J_{n}^{k}(\omega), \omega\right) d P_{x}(\omega) .
$$

Since we have shown that (5.4) implies (5.1), therefore for any $A \in \mathscr{F}_{\infty}, B \in \mathscr{M}_{\mathrm{J}_{n}^{k}}$ and $x \in E$,

$$
P_{x}(A \cap B)=\int_{B} Y_{A}\left(J_{n}^{k}(\omega), \omega\right) d P_{x}(\omega)
$$

In particular for an arbitrary stopping time $T$,

$$
P_{x}\left(T=J_{n}^{k}\right)=\int_{\left\{T=J_{n}^{k}\right\}} Y_{\left\{T=J_{n}^{k}\right\}}\left(J_{n}^{k}(\omega), \omega\right) d P_{x}(\omega)
$$


and therefore

$$
Y_{\left\{T=J_{n}^{k}\right\}}\left(J_{n}^{k}(\omega), \omega\right)=1, \quad P_{x} \text {-almost surely on }\left\{T=J_{n}^{k}\right\} .
$$

We now proceed to prove (5.4) for an arbitrary stopping time $T$ when $B \subset F$. If $\omega \in\left\{T=J_{n}^{k}\right\}$, then

$$
A_{0}\left(J_{n}^{k}(\omega), \omega\right)=\left(A_{0} \cap\left\{T=J_{n}^{k}\right\}\right)\left(J_{n}^{k}(\omega), \omega\right)+\left(A_{0} \cap\left\{T>J_{n}^{k}\right\}\right)\left(J_{n}^{k}(\omega), \omega\right) .
$$

Then (5.6) implies that

$$
\begin{aligned}
Y_{A_{0}}(T(\omega), \omega) & =P_{X(T(\omega))}\left(A_{0} \cap\left\{T=J_{n}^{k}\right\}\right)\left(J_{n}^{k}(\omega), \omega\right) \\
& =P_{X(T(\omega))}\left(X\left(t_{1}\right) \in \Gamma_{1}, \ldots, X\left(t_{n}\right) \in \Gamma_{n}\right) \cdot \chi_{C},
\end{aligned}
$$

$P_{x}$-almost surely on $\left\{T=J_{n}^{k}\right\}$. A similar result holds for $\{T=\zeta\} \cap F$. Hence it is easy to verify that

$$
\int_{B \cap C \cap F} P_{X(T(\omega))}\left(X\left(t_{1}\right) \in \Gamma_{1}, \ldots, X\left(t_{n}\right) \in \Gamma_{n}\right) d P_{x}(\omega)=\int_{B \cap F} Y_{A_{0}}(T(\omega), \omega) d P_{x}(\omega) .
$$

Proposition 2.9.1 implies that there is an increasing sequence of stopping times $\left\{S_{n}\right\}, S_{n} \leqq T$ for all $n$, such that:

(5.8) if $Q \equiv\left\{S_{n}<T\right.$ for all $\left.n, \lim _{n \rightarrow \infty} S_{n}=T\right\}$, then $P_{x}(Q \cap H)=P_{x}(H)$.

Let $S_{\infty} \equiv \lim _{n \rightarrow \infty} S_{n}$ and $Q^{\prime} \equiv\left\{S_{n}<S_{\infty}\right.$ for all $\left.n\right\}$.

We next prove (5.1) for the case $T \equiv S_{\infty}$. If $\omega \in Q^{\prime}$ and $\omega^{\prime} \sim \omega\left(R_{S_{\infty}}\right)$, then $S_{n}\left(\omega^{\prime}\right)=S_{n}(\omega)$ for all $n$ (cf. Proposition 4.2) and consequently $S_{\infty}\left(\omega^{\prime}\right)=S_{\infty}(\omega)$. If $\omega \in Q^{\prime}$ and if $A_{0}$ is defined by (5.2) (with $T \equiv S_{\infty}$ ), then

$$
\begin{aligned}
A_{0}\left(S_{\infty}(\omega), \omega\right) & =\left\{X\left(t_{1}\right) \in \Gamma_{1}, \ldots, X\left(t_{n}\right) \in \Gamma_{n}\right\} \cap\left\{X(0)=X\left(S_{\infty}(\omega)\right)\right\} \quad \text { if } \omega \in C \\
& =\varnothing \quad \text { if } \omega \notin C,
\end{aligned}
$$

and therefore

$$
Y_{A_{0}}\left(S_{\infty}(\omega), \omega\right)=P_{X\left(S_{\infty}(\omega)\right)}\left(X\left(t_{1}\right) \in \Gamma_{1}, \ldots, X\left(t_{n}\right) \in \Gamma_{n}\right) \chi_{C} .
$$

Hence it is easy to verify that for any set $A \in \mathscr{F}_{\infty}$ and any set $B \in \mathscr{M}_{S_{\infty}}$ and any $x$ such that $P_{x}\left(B \cap Q^{\prime}\right)=P_{x}(B)$, then

$$
P_{x}(B \cap A)=\int_{B} Y_{A}\left(S_{\infty}(\omega), \omega\right) d P_{x}(\omega) .
$$

In particular for an arbitrary stopping time $T$, if $P_{x}\left(\left\{T=S_{\infty}\right\} \cap Q^{\prime}\right)=P_{x}\left(\left\{T=S_{\infty}\right\}\right)$, then

$$
P_{x}\left(\left\{T=S_{\infty}\right\}\right)=\int_{\left\{T=S_{\infty}\right\}} Y_{\left\{T=S_{\infty}\right\}}\left(S_{\infty}(\omega), \omega\right) d P_{x}(\omega)
$$

and therefore

(5.10) $P_{X\left(S_{\infty}(\omega)\right)}\left(\left\{T=S_{\infty}\right\}\left(S_{\infty}(\omega), \omega\right)\right)=1, \quad P_{x^{-}}$almost surely on $\left\{T=S_{\infty}\right\}$. 
We are now ready to prove (5.4) for an arbitrary stopping time $T$ when $B \subset H$. Let $\left\{S_{n}\right\}$ be chosen to satisfy (5.8). For $\omega \in \Omega$ let $T_{\omega}^{\prime}(\cdot)$ be the $\left(T, \omega, S_{\infty}(\omega)\right)$-relative stopping time. Then (5.10) implies that $P_{X\left(S_{\infty}(\omega)\right)}\left(T_{\omega}^{\prime}(\cdot)=0\right)=1, P_{x}$-almost surely on $H$. For $\omega \in \Omega$ let $T_{\omega}^{\prime \prime}(\cdot)$ be the $(T, \omega, T(\omega))$-relative stopping time. If $\omega \in Q$, then $S_{\infty}(\omega)=T(\omega)$ and therefore

$$
P_{X(T(\omega))}\left(T_{\omega}^{\prime \prime}(\cdot)=0\right)=1, \quad P_{x} \text {-almost surely on } H .
$$

But if $A_{0}$ is defined by (5.2) and if $\omega \in Q$, then

$$
\begin{aligned}
A_{0}(T(\omega), \omega)= & \left\{X\left(t_{1}\right) \in \Gamma_{1}, \ldots, X\left(t_{n}\right) \in \Gamma_{n}\right\} \\
& \cap\left\{T_{\omega}^{\prime \prime}(\cdot)=0\right\} \cap\{X(0)=X(T(\omega))\} \\
& \cup A^{\prime} \cap\left\{T_{\omega}^{\prime \prime}(\cdot)>0\right\} \quad \text { if } \omega \in C \\
= & A^{\prime \prime} \cap\left\{T_{\omega}^{\prime \prime}(\cdot)>0\right\} \quad \text { if } \omega \notin C
\end{aligned}
$$

for some $A^{\prime} \in \mathscr{F}_{\infty}$ and $A^{\prime \prime} \in \mathscr{F}_{\infty}$. Thus (5.11) implies that

$$
Y_{A_{0}}(T(\omega), \omega)=\chi_{C} P_{X(T(\omega))}\left(X\left(t_{1}\right) \in \Gamma_{1}, \ldots, X\left(t_{n}\right) \in \Gamma_{n}\right),
$$

$P_{x}$-almost surely on $H$. Hence for $A_{0}$ defined by (5.2),

$$
\int_{B \cap H} Y_{A_{0}}(T(\omega), \omega) d P_{x}(\omega)=\int_{C \cap B \cap H} P_{X(T(\omega))}\left(X\left(t_{1}\right) \in \Gamma_{1}, \ldots, X\left(t_{n}\right) \in \Gamma_{n}\right) d P_{x}(\omega)
$$

and the proof is complete.

Corollary 1. If $A \in \mathscr{M}_{T+t}$, then except for a set of $\omega$ of $P_{x}$-probability zero $A(T(\omega), \omega) \supset B(\omega)$ such that $B(\omega) \in \mathscr{M}_{t}$ and such that $P_{X(T(\omega))}(A(T(\omega), \omega)-B(\omega))=0$.

Proof. The proof follows immediately from (5.6), (5.7), (5.11), and (5.12).

CoRollaRY 2. If $t<T\left(\omega_{0}\right)$, then $Y_{A}\left(t, \omega_{0}\right)=\int Y_{A}\left(T\left(\omega^{\prime}\right), \omega^{\prime}\right) d P_{X\left(t, \omega_{0}\right)}^{*}\left(\omega^{\prime}\right)$, where $P_{X\left(t, \omega_{0}\right)}^{*}$ is the measure on $\mathscr{F}_{\infty}$ defined by

$$
P_{X\left(t, \omega_{0}\right)}^{*}(A) \equiv P_{X\left(t, \omega_{0}\right)}\left(A\left(t, \omega_{0}\right)\right), \quad A \in \mathscr{F}_{\infty} .
$$

Proof. Let $T^{\prime}$ be the $\left(T, \omega_{0}, t\right)$-relative stopping time. Then Theorem 5.1 implies that

$$
\begin{aligned}
P_{X\left(t, \omega_{0}\right)}\left(A\left(t, \omega_{0}\right)\right) & =\int Y_{A\left(t, \omega_{0}\right)}\left(T^{\prime}\left(\omega^{\prime}\right), \omega^{\prime}\right) d P_{X\left(t, \omega_{0}\right)}\left(\omega^{\prime}\right) \\
& =\int Y_{A}\left(T\left(\omega^{\prime}\right), \omega^{\prime}\right) d P_{X\left(t, \omega_{0}\right)}^{*}\left(\omega^{\prime}\right) .
\end{aligned}
$$

The last equality is an immediate consequence of the definitions of $T^{\prime}$ and $P_{X\left(t, \omega_{0}\right)}^{*}$

REMARKS 5.1. Since the $\sigma$-fields $\left(\mathscr{M}_{t}\right)$ are right continuous, the process $Y_{A+}(t, \omega)$ $\equiv \lim _{s \downarrow t} Y_{A}(s, \omega)$ is a right continuous modification of the martingale $Y_{A}(t, \cdot)$ (refer to P. A. Meyer [13, Chapter 6, T 4]). In fact Professer P. A. Meyer has proved in a private communication that $Y_{A}(\cdot, \cdot)$ is actually well measurable [13, 
Chapter 8] and therefore [13, Chapter 8, T 20] it is easy to verify that $Y_{A}(\cdot, \omega)=$ $Y_{A^{+}}(\cdot, \omega) P_{x^{-a l m o s t}}$ surely for each $x$. However we make no use of this property in this paper.

5.2. From another point of view $Y .(t, \cdot)$ can be interpreted as a regular conditional probability with respect to the $\sigma$-field $\mathscr{M}_{t}$. The fact that $Y .(t, \omega)$ is a regular conditional probability, that is, for each $\omega \in \Omega, Y .(t, \omega)$ is a probability measure, follows from Proposition 4.4.

5.3. If $A$ is a set of the form $\left\{X_{T+s} \in \Gamma\right\}, \Gamma \in \mathscr{E}, s \geqq 0$, then Theorem 5.1 reduces to the usual statement of the strong Markov property. If $A \in \mathscr{M}_{T}^{*}$, then Theorem 5.1 reduces to a result of E. B. Dynkin [7, Theorem 3.11]. Hence Theorem 5.1 can be thought of as an extension of the usual strong Markov property to sets which need not belong to $\mathscr{M}_{T}^{*}$. Theorem 5.1 is also analogous to the Fubini theorem for product measures (applied to indicator functions of sets) and thus could also be interpreted as a Fubini-Markov theorem.

6. Nonequivalent pairs of Markov processes. Let

$$
\pi_{1}=\left(\Omega, \mathscr{F}_{\infty},\left(P_{x}^{1}\right)_{x \in E_{\Delta}},\left(X_{t}\right)_{t \in[0, \infty]}\right) \quad \text { and } \quad \pi_{2}=\left(\Omega, \mathscr{F}_{\infty},\left(P_{x}^{2}\right)_{x \in E_{\Delta}},\left(X_{t}\right)_{t \in[0, \infty]}\right)
$$

be a pair of Hunt processes which are instantaneously equivalent. It is assumed that for some $x_{0} \in E$ and $A_{0} \in \mathscr{F}_{\infty}, P_{x_{0}}^{1}\left(A_{0}\right)=0, P_{x_{0}}^{2}\left(A_{0}\right)=a_{0}>0$, that is, $A_{0}$ is a $\left(\pi_{1}, \pi_{2}, X_{0}\right)$ singularity. $Y_{A}^{1}(\cdot, \cdot)$ and $Y_{A}^{2}(\cdot, \cdot)$ denote the martingales defined in $\S 5$ for $\pi_{1}$ and $\pi_{2}$ respectively. For any real number $s$, let $\mathscr{R}_{s} \equiv\{r-s: r \geqq s, r$ rational $\}$.

Proposition 6.1. For each $s \in[0, \infty)$ and $A \in \mathscr{F}_{\infty}$,

$$
\begin{aligned}
T_{A}^{s}(\omega) & \equiv \inf \left\{t: t \in \mathscr{R}_{s}, Y_{A}^{1}(t, \omega)>0\right\} \\
& \equiv+\infty \quad \text { if }\left\{t: t \in \mathscr{R}_{s}, Y_{A}^{1}(t, \omega)>0\right\}=\varnothing .
\end{aligned}
$$

Then for each $s \in[0, \infty), T_{A}^{s}(\cdot)$ is a stopping time.

Proof. $\left\{\omega: T_{A}^{s}(\omega)<t\right\}=\bigcup_{r \in[0, t) \cap \mathscr{R}_{s}}\left\{\omega: Y_{A}^{1}(r, \omega)>0\right\}$. But Proposition 4.4 implies that $\left\{\omega: Y_{A}^{1}(r, \omega)>0\right\} \in \mathscr{M}_{t}$ if $r<t$ and therefore $T_{A}^{s}$ is a stopping time with respect to the $\sigma$-fields $\left(\mathscr{M}_{t}\right)_{t \in[0, \infty]}$.

Proposition 6.2. For any $s \in[0, \infty)$ and $A \in \mathscr{F}_{\infty}$,

(i) if $P_{x}^{1}(A)=0$, then $P_{x}^{1}\left(T_{A}^{s}<\infty\right)=0$, and

(ii) if $P_{x}^{1}(A)>0$, then $P_{x}^{1}\left(T_{A}^{s}=0\right)=1$.

Proof. (i) Since $\left\{T_{A}^{s}<\infty\right\}=\bigcup_{r \in \mathscr{R}_{s}}\left\{Y_{A}^{1}(r, \omega)>0\right\}, P_{x}^{1}\left(T_{A}^{s}<\infty\right)>0$ implies that for some $r \in \mathscr{R}_{s}, P_{x}\left(Y_{A}^{1}(r, \omega)>0\right)>0$. But then Theorem 5.1 implies that $P_{x}^{1}(A)>0$.

(ii) If $P_{x}^{1}\left(T_{A}^{s}=0\right)=0$, then for a sequence $\left\{r_{i}\right\}$ of elements of $\mathscr{R}_{s}$ such that $r_{i} \downarrow 0$ as $i \rightarrow \infty$, Theorem 5.1 implies that for each $i$

$$
P_{x}^{1}(A)=E_{x}^{1}\left(Y_{A}^{1}\left(r_{i}, \cdot\right)\right) \leqq P_{x}^{1}\left(T_{A}^{s} \leqq r_{i}\right) .
$$

Since $P_{x}^{1}\left(T_{A}^{s} \leqq r_{i}\right) \rightarrow 0$ as $i \rightarrow \infty$, therefore $P_{x}^{1}(A)=0$. Since $\left\{T_{A}^{s}=0\right\} \in \mathscr{M}_{0}$, the result then follows from the zero-one law (Proposition 2.7.1). 
Remarks. 6.1. For $s=0$ we let $T_{A}^{0}=T_{A}$. Since $\pi_{1}$ and $\pi_{2}$ are assumed to be instantaneously equivalent and $P_{x_{0}}^{1}\left(A_{0}\right)=0$, then Proposition 6.2 implies that $P_{x_{0}}^{1}\left(T_{A_{0}}=0\right)=P_{x_{0}}^{2}\left(T_{A_{0}}=0\right)=0$.

6.2. Let $S$ be a stopping time and let $A \in \mathscr{M}_{S}$. It is easy to verify that for each $\omega \in \Omega$ either $T_{A}(\omega) \leqq S(\omega)$ or $T_{A}(\omega)=\infty$. In fact if $\infty>t>S(\omega)$, then $A(t, \omega)$ is either $\Omega$ or $\varnothing$ depending on whether $\omega \in A$ or not (cf. Proposition 4.1).

We next state and prove three technical results which are used in $\S 8$.

Proposition 6.3. Let $S$ be an $\left(\mathscr{M}_{t}\right)$-stopping time such that $S \leqq T_{A_{0}}$ and such that $P_{x_{0}}^{2}\left(A_{0} \cap\left\{S<T_{A_{0}}\right\}\right)=P_{x_{0}}^{2}\left(A_{0}\right)$. Then $P_{x_{0}}^{2}\left(A_{0} \cap\left\{Y_{A_{0}}^{1}(S(\omega), \omega)>0\right\}\right)=0$.

Proof. If $Y_{A_{0}}^{1}(S(\omega), \omega)>0$, then $P_{X(S(\omega))}^{1}\left(A_{0}(S(\omega), \omega)\right)>0$ and Proposition 6.2 implies that $P_{X(S(\omega))}^{1}\left(T_{A_{0}(S(\omega), \omega)}^{S(\omega)}=0\right)=1$. Since $\pi_{1}$ and $\pi_{2}$ are instantaneously equivalent, this implies that $P_{X(S(\omega))}^{2}\left(T_{A_{0}(S(\omega), \omega)}^{S(\omega)}=0\right)=1$. If $Y_{A_{0}}^{1}(S(\omega), \omega)=0$, then $P_{X(S(\omega))}^{1}\left(A_{0}(S(\omega), \omega)\right)=0$ and Proposition 6.2 implies that

$$
P_{X(S(\omega))}^{1}\left(T_{A_{0}(S(\omega), \omega)}^{S(\omega)}>0\right)=P_{X(S(\omega))}^{2}\left(T_{A_{0}(S(\omega), \omega)}^{S(\omega)}>0\right)=1 .
$$

If $\omega^{\prime} \sim \omega\left(R_{S(\omega)}\right)$, then Proposition 4.2 implies that $S\left(\omega^{\prime}\right) \geqq S(\omega)$. Therefore $\left\{T_{A_{0}}>S\right\}(S(\omega), \omega) \subset\left\{\omega^{\prime}: T_{A_{0}(S(\omega), \omega)}^{S(\omega)}\left(\omega^{\prime}\right)>0\right\}$. But Theorem 5.1 implies that

$$
\begin{aligned}
P_{x_{0}}^{2}\left(A_{0}\right) & =P_{x_{0}}^{2}\left(A_{0} \cap\left\{T_{A_{0}}>S\right\}\right) \\
& =\int P_{X(S(\omega))}^{2}\left[\left\{T_{A_{0}}>S\right\}(S(\omega), \omega)\right] \cdot P_{X(S(\omega))}^{2}\left(A_{0}(S(\omega), \omega)\right) d P_{x_{0}}^{2}(\omega) \\
& \leqq \int P_{X(S(\omega))}^{2}\left[T_{A_{0}(S(\omega), \omega)}^{S(\omega)}\left(\omega^{\prime}\right)>0\right] \cdot P_{X(S(\omega))}^{2}\left(A_{0}(S(\omega), \omega)\right) d P_{x_{0}}^{2}(\omega) \\
& =P_{x_{0}}^{2}\left(A_{0} \cap\left\{Y_{A_{0}}^{1}(S(\cdot), \cdot)=0\right\}\right) \quad \text { by }(6.1) .
\end{aligned}
$$

Hence $P_{x_{0}}^{2}\left(A_{0} \cap\left\{Y_{A_{0}}^{1}(S(\cdot), \cdot)>0\right\}\right)=0$ and the proof is complete.

Proposition 6.4. $P_{x_{0}}^{2}\left(A_{0} \cap B_{0}\right)=P_{x_{0}}^{2}\left(A_{0}\right)$ where $B_{0} \equiv\left\{Y_{A_{0}}^{1}\left(T_{A_{0}}(\cdot), \cdot\right)>0\right\}$.

Proof. As in the proof of Theorem 5.1 let

$$
H \equiv\left\{X_{T_{A_{0}}}^{-}=X_{T_{A_{0}}}\right\} \cap\left\{T_{A_{0}}<\infty\right\} \text { and } F=\left\{X_{T_{A_{0}}}^{-} \neq X_{T_{A_{0}}}\right\} \cap\left\{T_{A_{0}}<\infty\right\} \text {. }
$$

It is easy to verify that $P_{x_{0}}^{2}\left(B_{0} \cap A_{0} \cap\left\{T_{A_{0}}=\infty\right\}\right)=P_{x_{0}}^{2}\left(A_{0} \cap\left\{T_{A_{0}}=\infty\right\}\right)$. To prove that $P_{x_{0}}^{2}\left(B_{0} \cap A_{0} \cap F\right)=P_{x_{0}}^{2}\left(A_{0} \cap F\right)$ it suffices to show that

$$
P_{x_{0}}^{2}\left(B_{0} \cap A_{0} \cap F \cap\left\{T_{A_{0}}=\zeta\right\}\right)=P_{x_{0}}^{2}\left(A_{0} \cap F \cap\left\{T_{A_{0}}=\zeta\right\}\right)
$$

and that $P_{x_{0}}^{2}\left(B_{0} \cap A_{0} \cap\left\{T_{A_{0}}=J_{n}^{k}\right\}\right)=P_{x_{0}}^{2}\left(A_{0} \cap\left\{T_{A_{0}}=J_{n}^{k}\right\}\right)$ for $k, n=1,2,3, \ldots$, where the $J_{n}^{k} ; k, n=1,2,3, \ldots$, are defined as in the proof of Theorem 5.1. Noting that $\left\{T_{A_{0}}>J_{n}^{k}\right\}\left(J_{n}^{k}(\omega), \omega\right) \in \mathscr{M}_{0}$, we can easily verify that Theorem 5.1 implies

$$
\begin{aligned}
P_{x_{0}}^{2}\left(A_{0} \cap\left\{T_{A_{0}}=J_{n}^{k}\right\}\right)= & \int_{\left(T_{A_{0}=J_{n}^{k}}^{k}\right\}} P_{X\left(J_{n}^{k}(\omega)\right)}^{2}\left(A_{0}\left(J_{n}^{k}(\omega), \omega\right)\right) \\
& \cdot P_{X\left(J_{n}^{k}(\omega)\right)}^{2}\left(\left\{T_{A_{0}}=J_{n}^{k}\right\}\left(J_{n}^{k}(\omega), \omega\right)\right) \cdot d P_{x_{0}}^{2}(\omega)
\end{aligned}
$$


But Proposition 6.2 and the instantaneous equivalence of $\pi_{1}$ and $\pi_{2}$ imply that for $\omega \in\left\{T_{A_{0}}=J_{n}^{k}\right\}$,

$$
P_{X\left(J_{n}^{k}(\omega)\right)}^{2}\left(\left\{T_{A_{0}}=J_{n}^{k}\right\}\left(J_{n}^{k}(\omega), \omega\right)\right)=P_{X\left(J_{n}^{k}(\omega)\right)}^{1}\left(\left\{T_{A_{0}}=J_{n}^{k}\right\}\left(J_{n}^{k}(\omega), \omega\right)\right)=\chi_{B_{0}} .
$$

Hence $P_{x_{0}}^{2}\left(A_{0} \cap\left\{T_{A_{0}}=J_{n}^{k}\right\}\right)=P_{x_{0}}^{2}\left(A_{0} \cap B_{0} \cap\left\{T_{A_{0}}=J_{n}^{k}\right\}\right)$. A similar result may be obtained for $(T=\zeta) \cap F$.

To prove that $P_{x_{0}}^{2}\left(A_{0} \cap B_{0} \cap H\right)=P_{x_{0}}^{2}(A \cap H)$, note that the proof of Theorem 5.1 (in particular (5.11)) implies that there is a set $Q \in \mathscr{F}_{\infty}$ such that

$$
P_{x_{0}}^{2}(H \cap Q)=P_{x_{0}}^{2}(H)
$$

and such that $P_{X\left(T_{A_{0}}(\omega)\right)}^{2}\left(T_{A_{0}\left(T_{A_{0}}(\omega), \omega\right)}^{T_{A_{0}}(\omega)}=0\right)=1$ for each $\omega \in Q$. But Proposition 6.2 and the instantaneous equivalence of $\pi_{1}$ and $\pi_{2}$ imply that $P_{X\left(T_{A_{0}}(\omega)\right)}^{2}\left(T_{A_{0}\left(T_{A_{0}}(\omega), \omega\right)}^{T_{A}(\omega)}=0\right)=1$ if and only if $Y_{A_{0}}^{1}\left(T_{A_{0}}(\omega), \omega\right)>0$. Hence it follows that $P_{x_{0}}^{2}\left(A_{0} \cap B_{0} \cap H\right)=$ $P_{x_{0}}^{2}\left(A_{0} \cap H\right)$.

REMARK 6.3. It is easy to verify that $P_{x_{0}}^{1}\left(B_{0}\right)=0, P_{x_{0}}^{2}\left(B_{0}\right) \geqq a_{0}$ and that $B_{0} \in \mathscr{M}_{T_{A_{0}}}$. Furthermore, $P_{x_{0}}^{2}\left(A_{0}-B_{0}\right)=P_{x_{0}}^{1}\left(A_{0}-B_{0}\right)=0$. Finally, Theorem 5.1, Corollary 2 implies that $T_{B_{0}} \geqq T_{A_{0}}$. Remark 6.2 implies that either $T_{B_{0}} \leqq T_{A_{0}}$ or $T_{B_{0}}=\infty$, and therefore

$$
\begin{aligned}
T_{B_{0}}(\omega) & =T_{A_{0}}(\omega) & & \text { if } Y_{A_{0}}^{1}\left(T_{A_{0}}(\omega), \omega\right)>0 \\
& =\infty & & \text { if } Y_{A_{0}}^{1}\left(T_{A_{0}}(\omega), \omega\right)=0 .
\end{aligned}
$$

Proposition 6.5. There is a set $B_{0}^{\prime} \supset A_{0}, B_{0}^{\prime} \in \mathscr{M}_{T_{A_{0}}}$, such that $T_{A_{0}}=T_{B_{0}^{\prime}}$.

Proof. For any rational $r \in[0,1 / n]$, let $T_{r}^{n}, n=1,2,3, \ldots$, be the stopping time defined by

$$
T_{r}^{n}=\sum_{i=-1}^{\infty} \chi_{\left\{r+i / n \leqq T_{A 0}<r+(i+1) / n\right)} \cdot(r+(i+1) / n)+\chi_{\left(T_{A 0}=\infty\right)} \cdot(\infty) .
$$

Note that $T_{A_{0}}+1 / n \geqq T_{r}^{n} \geqq T_{A_{0}}$ and that the definition of $T_{A_{0}}$ implies that

$$
A_{0} \subset \bigcup_{r \in[0,1 / n] \cap \mathscr{R}_{0}}\left\{Y_{A_{0}}^{1}\left(T_{r}^{n}(\omega), \omega\right)>0\right\} \equiv B_{n} .
$$

Hence $A_{0} \subset B_{0}^{\prime} \equiv \bigcap_{k=1}^{\infty} \bigcup_{n=k}^{\infty} B_{n}$. But since $B_{n} \in \mathscr{M}_{T_{A_{0}}+1 / n}, B_{0}^{\prime} \in \mathscr{M}_{T_{A_{0}}}$. Finally, Remark 6.2 implies that $T_{B_{0}^{\prime}} \leqq T_{A_{0}}$ or $=+\infty$ and Theorem 5.1, Corollary 2 implies that $T_{B_{0}^{\prime}} \geqq T_{A_{0}}$; in fact for $t<T_{A_{0}}(\omega), Y_{B_{0}^{\prime}}^{1}(t, \omega)>0$ if and only if $Y_{A_{0}}^{1}(t, \omega)>0$. But the definition of $B_{0}^{\prime}$ implies that if $T_{A_{0}}(\omega)<\infty$ then $T_{B_{0}^{\prime}(\omega)}<\infty$ so that $T_{A_{0}}=T_{B_{0}^{\prime}}$. Note that this implies that $P_{x_{0}}^{1}\left(B_{0}^{\prime}\right)=0$.

7. The canonical singularities. Let $\pi_{1}$ and $\pi_{2}$ be a pair of Hunt processes. For $t>0$ and $0<\alpha \leqq 1$ let

$$
K_{t}^{\alpha} \equiv\left\{x \in E: \text { for some } C \in \mathscr{F}_{t}, P_{x}^{1}(C)=0, P_{x}^{2}(C) \geqq \alpha\right\} .
$$

For a fixed value of $\alpha, K_{t}^{\alpha}$ decreases as $t$ decreases.

Proposition 7.1. For each $0<\alpha \leqq 1$ and $t>0, K_{t}^{\alpha}$ is an $\mathscr{E}$-measurable set. 
Proof. Proposition 2.2.1 implies that there exists a countable algebra $\mathscr{C}_{t}$ of sets which generates $\mathscr{F}_{t}$, say $\mathscr{C}_{t}=\left\{B_{m}: m=1,2,3, \ldots\right\}$. If

$$
K_{t}^{\alpha, n} \equiv \bigcup_{m=1}^{\infty}\left\{x: P_{x}^{1}\left(B_{m}\right)<1 / 2^{n}, P_{x}^{2}\left(B_{m}\right) \geqq \alpha-1 / 2^{n}\right\}, \quad \text { then } K_{t}^{\alpha, n} \in \mathscr{E} .
$$

It is easy to verify that $K_{t}^{\alpha} \subset \bigcap_{n=1}^{\infty} K_{t}^{\alpha, n}$ by the approximation theorem for measures. On the other hand if $x \in \bigcap_{n=1}^{\infty} K_{t}^{\alpha, n}$, then for each $n$ there is a set $A_{n} \in \mathscr{F}_{t}$ such that $P_{x}^{1}\left(A_{n}\right)<1 / 2^{n}$ and $P_{x}^{2}\left(A_{n}\right) \geqq \alpha-1 / 2^{n}$. But then $P_{x}^{1}\left(\bigcap_{k=1}^{\infty} \bigcup_{n=k}^{\infty} A_{n}\right)=0$ and

$$
P_{x}^{2}\left(\bigcap_{k=1}^{\infty} \bigcup_{n=k}^{\infty} A_{n}\right) \geqq \alpha
$$

so that $x \in K_{t}^{\alpha}$. Hence $K_{t}^{\alpha}=\bigcap_{n=1}^{\infty} K_{t}^{\alpha, n}$ and therefore $K_{t}^{\alpha} \in \mathscr{E}$.

Proposition 7.2. $\pi_{1}$ and $\pi_{2}$ are instantaneously equivalent if and only if $\bigcap_{t>0} K_{t}^{\alpha}=\varnothing$ for each $\alpha>0$.

Proof. If $x \in \bigcap_{t>0} K_{t}^{\alpha}$, then for any positive integer $n$ there is a set $A_{n} \in \mathscr{F}_{1 / n}$ such that $P_{x}^{1}\left(A_{n}\right)=0$ and $P_{x}^{2}\left(A_{n}\right) \geqq \alpha$. But then $A \equiv \bigcap_{k=1}^{\infty} \bigcup_{n=k}^{\infty} A_{n} \in \mathscr{M}_{0}$ and $P_{x}^{1}(A)=0$ and $P_{x}^{2}(A) \geqq \alpha$. Hence $\pi_{1}$ and $\pi_{2}$ are not instantaneously equivalent. On the other hand if $\pi_{1}$ and $\pi_{2}$ are not instantaneously equivalent then there is a set $A \in \mathscr{M}_{0}$ and $x \in E$ such that $P_{x}^{1}(A)=0, P_{x}^{2}(A)=1$ and therefore $x \in K_{t}^{\alpha}$ for all $t>0$ and $\alpha \leqq 1$.

To each set $K_{t}^{\alpha}, t>0,0<\alpha \leqq 1$, we now construct a canonical singular event $\hat{K}_{t}^{\alpha}$. For $B_{m} \in \mathscr{C}_{t}, m=1,2,3, \ldots$, and each positive integer $n$, let

$$
K_{m}^{n} \equiv\left\{x: P_{x}^{1}\left(B_{m}\right)<1 / 2^{n}, P_{x}^{2}\left(B_{m}\right) \geqq \alpha-1 / 2^{n}\right\} \cap K_{t}^{\alpha},
$$

and

$$
\begin{aligned}
\hat{K}^{n} \equiv\left(\left\{X(0) \in K_{1}^{n}\right\} \cap B_{1}\right) & \cup\left(\left\{X(0) \in K_{2}^{n}-K_{1}^{n}\right\} \cap B_{2}\right) \cup \cdots \\
& \cup\left(\left\{X(0) \in\left(K_{r}^{n}-\bigcup_{s=1}^{r-1} K_{s}^{n}\right)\right\} \cap B_{r}\right) \cup \cdots,
\end{aligned}
$$

and let $\hat{K}_{t}^{\alpha} \equiv \bigcap_{k=1}^{\infty} \bigcup_{n=k}^{\infty} \hat{K}^{n}$. Since for each $m, P_{x}\left(B_{m}\right)$ is measurable (cf. 2.3.2), it is easy to verify that $\hat{K}_{t}^{\alpha} \in \mathscr{F}_{t}$. Moreover $P_{x}^{1}\left(\hat{K}^{n}\right) \leqq 1 / 2^{n}$ for each $x \in E$ and $P_{x}^{2}\left(\hat{K}^{n}\right)$ $\geqq \alpha-1 / 2^{n}$ for each $x \in K_{t}^{\alpha}$. Hence $P_{x}^{1}\left(\hat{K}_{t}^{\alpha}\right)=0$ for each $x \in E$ and $P_{x}^{2}\left(\hat{K}_{t}^{\alpha}\right) \geqq \alpha$ for each $x \in K_{t}^{\alpha}$.

8. Classification of singularities. In this section we classify the types of singularities which can occur for a pair $\left(\pi_{1}, \pi_{2}\right)$ of Hunt processes.

8.1. Types of singularities. An event $A \in \mathscr{F}_{\infty}$ is a $\left(\pi_{1}, \pi_{2}, X_{0}\right)$ germ field singularity if $A \in \mathscr{M}_{0}, P_{x_{0}}^{1}(A)=0$ and $P_{x_{0}}^{2}(A)>0$.

$A \in \mathscr{F}_{\infty}$ is a $\left(\pi_{1}, \pi_{2}, x_{0}\right)$ singularity of tail type if $P_{x_{0}}^{1}(A)=0, P_{x_{0}}^{2}(A)>0$ and $Y_{A}^{1}(t)=0, P_{x_{0}}^{2}$-almost surely for all $t \in[0, \infty)$.

$A \in \mathscr{F}_{\infty}$ is a $\left(\pi_{1}, \pi_{2}, x_{0}\right)$ tail field singularity if $A$ belongs to the tail field $\mathscr{F}_{\infty}^{*} \equiv \bigcap_{t>0} \mathscr{F}_{t}^{*}$, and $A$ is a $\left(\pi_{1}, \pi_{2}, x_{0}\right)$ tail type singularity.

$A \in \mathscr{F}_{\infty}$ is a $\left(\pi_{1}, \pi_{2}, x_{0}\right)$ jump singularity at $T$ if: 
(i) $T$ is a stopping time which is totally inaccessible in the weak sense on $A$ with respect to $P_{x_{0}}^{2}$ and $A \in \mathscr{M}_{T}$,

(ii) for any stopping time $S$ such that $P_{x_{0}}^{2}(A \cap\{S<T\})=P_{x_{0}}^{2}(A), Y_{A}^{1}(S)=0$, $P_{x_{0}}^{2}$-almost surely on $A$, and

(iii) $P_{x_{0}}^{1}(A)=0, P_{x_{0}}^{2}(A)>0$.

$A \in \mathscr{F}_{\infty}$ is a $\left(\pi_{1}, \pi_{2}, x_{0}\right)$ pseudo local singularity at $T$ if $T$ is a stopping time and there is an increasing sequence of stopping times $\left\{T_{n}\right\}$ such that:

(i) $T_{n}<T<\infty$ for every $n, P_{x_{0}}^{2}$-almost surely on $A$,

(ii) $\lim _{n \rightarrow \infty} T_{n}=T, P_{x_{0}}^{2}$-almost surely on $A$,

(iii) $A \in \mathscr{M}_{T}, P_{x_{0}}^{1}(A)=0, P_{x_{0}}^{2}(A)>0$ and

(iv) for every $n, Y_{A}^{1}\left(T_{n}\right)=0, P_{x_{0}}^{2}$-almost surely on $A$. In such case it is easy to verify that $T_{A}(\omega)$ is either $T(\omega)$ or $\infty, P_{x_{0}}^{2}$-almost surely on $A$.

Let $\left\{T_{n}\right\}$ be an increasing sequence of stopping times, such that $T_{n} \leqq T$ for each $n$. The $\sigma$-field $\mathscr{M}_{\left\{T_{n}\right\}, T}$ is defined to be $\bigcap_{n=1}^{\infty} \mathscr{M}_{T_{n}}^{*} \cap \mathscr{M}_{T}$.

$A \in \mathscr{F}_{\infty}$ is said to be local at a stopping time $T$ with respect to $P_{x_{0}}^{2}$ if there is an increasing sequence of stopping times $\left\{T_{n}\right\}$ such that $T_{n} \leqq T$ for each $n$ and such that:

(i) $T_{n}<T$ for each $n$ and $\lim _{n \rightarrow \infty} T_{n}=T<\infty$, both $P_{x_{0}}^{2}$-almost surely on a set $B$ which contains $A$,

(ii) $T_{n+1}$ is measurable with respect to $\mathscr{M}_{T_{n}}^{*}$, and

(iii) $A$ belongs to the local $\sigma$-field $\mathscr{M}_{\left\{T_{n}\right\}, T}$.

REMARK 8.1.1. It can be shown that the $P_{x_{0}}^{2}$-completion of the $\sigma$-field $\mathscr{M}_{\left\{T_{n}\right\}, T}$ restricted to the set $B$ is independent of the choice of the sequence $\left\{T_{n}\right\}$ as long as $\left\{T_{n}\right\}$ satisfies (i) and (ii). $\mathscr{M}_{\left\{T_{n}\right\}, T}$ is known as the local $\sigma$-field at $T$ with respect to $P_{x_{0}}^{2}$. Loosely speaking, events in $\mathscr{M}_{\left\{T_{n}\right\}, T}$ depend only on the behavior of the paths near $T$.

$A \in \mathscr{F}_{\infty}$ is a $\left(\pi_{1}, \pi_{2}, x_{0}\right)$ local singularity at $T$ if $A$ is a local event at the stopping time $T$ with respect to $P_{x_{0}}^{2}$ and $A$ is a $\left(\pi_{1}, \pi_{2}, x_{0}\right)$ pseudo local singularity at $T$.

8.2. A property of pseudo local singularities. Let $G$ be a $\left(\pi_{1}, \pi_{2}, x_{0}\right)$ pseudo local singularity such that $P_{x_{0}}^{2}(G)=a>0$. Then there is an increasing sequence of stopping times $\left\{T_{n}\right\}$ such that $T_{n}<T_{G}<\infty$ for all $n$ and $\lim _{n \rightarrow \infty} T_{n}=T_{G}$, both $P_{x_{0}}^{2}$-almost surely on $G$. Without loss of generality the sequence of stopping times $\left\{T_{n}\right\}$ can be chosen so that for a given $\varepsilon, 0<\varepsilon<a / 2, P_{x_{0}}^{2}\left(G \cap\left\{T_{G} \geqq T_{n}+1 / n\right\}\right)<\varepsilon / 2^{n}$. Let $G_{n} \equiv\left\{T_{G}<T_{n}+1 / n\right\} \cap G$. Then $G_{n} \in \mathscr{M}_{T_{n}+1 / n}$ (refer to P. A. Meyer [13, Chapter 4, T 39]). Corollary 1 of Theorem 5.1 implies that there is a set $Q \in \mathscr{F}_{\infty}$ such that $P_{x_{0}}^{2}(G \cap Q)=P_{x_{0}}^{2}(G)$ and such that for every $n$ and $\omega \in Q$,

$$
G_{n}\left(T_{n}(\omega), \omega\right) \supset G_{n}^{+}(\omega) \text { with } G_{n}^{+}(\omega) \in \mathscr{M}_{1 / n},
$$

and such that $P_{X\left(T_{n}(\omega)\right)}^{2}\left(G_{n}\left(T_{n}(\omega), \omega\right)-G_{n}^{+}(\omega)\right)=0$. Then we have the following result.

Proposition 8.2.1. $P_{x_{0}}^{2}\left(G \cap\left(\bigcap_{n=1}^{\infty}\left\{X\left(T_{n}\right) \in K_{2 / n}^{b}\right\}\right)\right) \geqq b$ where $b \equiv(a-\varepsilon) / 2$. 
Proof. If $G_{\infty} \equiv \bigcap_{n=1}^{\infty} G_{n}$, then $P_{x_{0}}^{2}\left(G_{\infty}\right) \geqq a-\varepsilon$. Consider

$$
\begin{aligned}
N(\omega) & \equiv \min \left\{n: Y_{G_{\infty}}^{2}\left(T_{n}(\omega), \omega\right) \leqq b\right\} \\
& \equiv+\infty \quad \text { if }\left\{n: Y_{G_{\infty}}^{2}\left(T_{n}(\omega), \omega\right) \leqq b\right\}=\varnothing .
\end{aligned}
$$

Let $\tilde{T}(\omega) \equiv T_{N(\omega)}$ where $T_{\infty}(\omega) \equiv T_{G}(\omega)$. Since

$$
\{\tilde{T}<t\}=\bigcup_{n=1}^{\infty}\left\{T_{n}<t\right\} \cap\left\{Y_{G_{\infty}}^{2}\left(T_{n}\right) \leqq b, Y_{G_{\infty}}^{2}\left(T_{n-1}\right)>b, \ldots, Y_{G_{\infty}}^{2}\left(T_{1}\right)>b\right\},
$$

it follows that $\{\tilde{T}<t\} \in \mathscr{M}_{t}$ and therefore $\tilde{T}$ is a stopping time. But Theorem 5.1 implies that

$$
\begin{aligned}
2 b \leqq P_{x_{0}}^{2}\left(G_{\infty}\right)= & \int P_{X(\tilde{T}(\omega))}^{2}\left(G_{\infty}(\tilde{T}(\omega), \omega)\right) d P_{x_{0}}^{2}(\omega) \\
= & \int_{\left\{\tilde{T}=T_{G}\right\}} P_{X(\tilde{T}(\omega))}^{2}\left(G_{\infty}(\tilde{T}(\omega), \omega)\right) d P_{x_{0}}^{2}(\omega) \\
& +\int_{\left\{\tilde{T}<T_{G}\right\}} P_{X(\tilde{T}(\omega))}^{2}\left(G_{\infty}(\tilde{T}(\omega), \omega)\right) d P_{x_{0}}^{2}(\omega) \\
= & \int_{\left(Y_{G_{\infty}}^{2}(\tilde{T}(\omega), \omega)<b\right\}} Y_{G_{\infty}}^{2}(\tilde{T}(\omega), \omega) d P_{x_{0}}^{2}(\omega) \\
& +\int_{\left\{Y_{G_{\infty}}^{2}(\tilde{T}(\omega), \omega) \geqq b\right\}} Y_{G_{\infty}}^{2}(\tilde{T}(\omega), \omega) d P_{x_{0}}^{2}(\omega) .
\end{aligned}
$$

But then $\int_{\left\{Y_{G_{\infty}}^{2}(\tilde{T}(\omega), \omega) \geqq b\right\}} Y_{G_{\infty}}^{2}(\tilde{T}(\omega), \omega) d P_{x_{0}}^{2}(\omega) \geqq b$ and therefore

$$
P_{x_{0}}^{2}\left(G_{\infty} \cap\left\{\bigcap_{n=1}^{\infty} Y_{G_{\infty}}^{2}\left(T_{n}(\omega), \omega\right) \geqq b\right\}\right) \geqq b .
$$

Since for each $n$

$$
P_{x_{0}}^{2}\left(G_{\infty} \cap\left\{Y_{G_{\infty}}^{1}\left(T_{n}(\omega), \omega\right) \geqq b\right\}\right)=0,
$$

then in view of (8.2.1)

$$
P_{x_{0}}^{2}\left(G_{\infty} \cap\left(\bigcap_{n=1}^{\infty}\left\{X\left(T_{n}\right) \in K_{2 / n}^{b}\right\}\right)\right) \geqq b
$$

and the proof is complete.

COROLlARY. There exist compact sets $\widetilde{K}_{2 / n}^{b} \subset K_{2 / n}^{b}$ such that

$$
P_{x_{0}}^{2}\left(G_{\infty} \cap\left(\bigcap_{n=1}^{\infty}\left\{X\left(T_{n}\right) \in \tilde{K}_{2 / n}^{b}\right\}\right)\right) \geqq b / 2
$$

Proof. This follows immediately since the measures $P_{x_{0}}^{2}\left(X\left(T_{n}\right) \in \cdot\right)$ are inner regular on $\mathscr{E}_{\Delta}$.

In the case in which $C$ is a $\left(\pi_{1}, \pi_{2}, x_{0}\right)$ tail type singularity, a similar type of argument yields the following result. 
Proposition 8.2.2. If $C$ is $a\left(\pi_{1}, \pi_{2}, x_{0}\right)$ tail type singularity such that $P_{x_{0}}^{2}(C)=a$ and $s \in[0, \infty)$, then

$$
P_{x_{0}}^{2}\left(C \cap\left\{X(s) \in K_{\infty}^{a / 2}\right\}\right) \geqq a / 2 .
$$

In the case in which $D$ is a $\left(\pi_{1}, \pi_{2}, x_{0}\right)$ jump singularity, a similar type of argument yields the following result.

Proposition 8.2.3. If $D$ is $a\left(\pi_{1}, \pi_{2}, x_{0}\right)$ jump singularity at $T_{D}$ with

$$
P_{x_{0}}^{2}\left(D \cap\left\{S<T_{D}<S+t\right\}\right)=a^{\prime}>0
$$

for some $t \in[0, \infty)$, then

$$
P_{x_{0}}^{2}\left(X(S) \in K_{2 t}^{a^{\prime} / 2}\right) \geqq a^{\prime} / 2 .
$$

\subsection{A result on tail type singularities.}

THEOREM 8.3.1. If $\left(\pi_{1}, \pi_{2}\right)$ have a tail type singularity then they have a tail field singularity.

Proof. Let $C$ be a $\left(\pi_{1}, \pi_{2}, x_{0}\right)$ tail type singularity with $P_{x_{0}}^{2}(C)=a$. Then Proposition 8.2.2 implies that $P_{x_{0}}^{2}\left(C \cap\left\{X(s) \in K_{\infty}^{a / 2}\right\}\right) \geqq a / 2$ for every $s \in[0, \infty)$. But then $P_{x_{0}}^{2}\left(\theta_{s}^{-1} \hat{K}_{\infty}^{a / 2}\right) \geqq a^{2} / 4$ and $P_{x_{0}}^{1}\left(\theta_{s}^{-1} \hat{K}_{\infty}^{a / 2}\right)=0$. Moreover $\theta_{s}^{-1} \hat{K}_{\infty}^{a / 2} \in \mathscr{M}_{s}^{*}$. Hence if $C_{0} \equiv \bigcap_{n=1}^{\infty} \bigcup_{k=n}^{\infty} \theta_{k}^{-1} \hat{K}_{\infty}^{a / 2}$, then $C_{0} \in \mathscr{F}_{\infty}^{*}, P_{x_{0}}^{2}\left(C_{0}\right)>0$ and $P_{x_{0}}^{2}\left(C_{0}\right)=0$. Hence $C_{0}$ is a tail field singularity.

8.4. A result on local singularities.

THEOREM 8.4.1. If $\left(\pi_{1}, \pi_{2}\right)$ have a pseudo local singularity, then $\left(\pi_{1}, \pi_{2}\right)$ have a local singularity. Moreover for some $\alpha>0, K_{t}^{\alpha} \neq \varnothing$ for all $t>0$.

Proof. Let $G$ be a $\left(\pi_{1}, \pi_{2}, x_{0}\right)$ pseudo local singularity at a stopping time $T$ with $P_{x_{0}}^{2}(G)=a$. But then Proposition 8.2.1 implies that for $0<\varepsilon<a / 2$,

$$
P_{x_{0}}^{2}\left(\bigcap_{n=1}^{\infty}\left(X\left(T_{n}\right) \in \tilde{K}_{2 / n}^{b}\right) \cap G\right) \geqq b / 2
$$

where $b=(a-\varepsilon) / 2, \widetilde{K}_{2 / n}^{b}$ is a compact subset of $K_{2 / n}^{b}$ and $\left\{T_{n}\right\}$ is a sequence of stopping times such that $\lim _{n \rightarrow \infty} T_{n}=T, P_{x_{0}}^{2}$-almost surely on $G$. This implies that $K_{2 / n}^{b} \neq \varnothing$ for every positive integer $n$.

Consider the sequence of stopping times defined by:

$$
\begin{aligned}
& S_{1}^{b} \equiv 0, \\
& S_{n}^{b} \equiv S_{n-1}^{b}+S_{n} \circ \theta_{S_{n-1}^{b}} \quad \text { for } n=2,3, \ldots,
\end{aligned}
$$

where the $S_{n}, n=2,3, \ldots$, are defined as follows. If

$$
\begin{aligned}
\tilde{S}_{n} & \equiv \inf \left\{s: X_{s}(\omega) \in \tilde{K}_{2 / n}^{b}\right\}, \quad n=2,3, \ldots \\
& \equiv+\infty \quad \text { if the above set is empty, }
\end{aligned}
$$

then $\tilde{S}_{n}$ is an $\left(\tilde{\mathscr{M}}_{t}^{\pi}{ }^{2}\right)$-stopping time. We then let $S_{n}$ be an $\left(\mathscr{M}_{t}\right)$-stopping time chosen 
so that $P_{\mu}^{2}\left(\tilde{S}_{n} \neq S_{n}\right)=0$ where $\mu(\cdot) \equiv P_{x_{0}}^{2}\left(X\left(S_{n-1}^{b}\right) \in \cdot\right)$. Let $S_{0}^{b} \equiv \lim _{n \rightarrow \infty} S_{n}^{b}, \hat{S}_{n}^{b} \equiv \theta_{S_{n}^{b}}^{-1} \hat{K}_{2 / n}^{b}$ and $\hat{S}_{0}^{b} \equiv \bigcap_{k=1}^{\infty} \bigcup_{n=k}^{\infty} \hat{S}_{n}^{b}$. The event $\hat{S}_{0}^{b} \cap\left(S_{0}^{b}<\infty\right)$ is a local event and $P_{x_{0}}^{1}\left(\hat{S}_{0}^{b}\right)=0$. Moreover (8.4.1) implies that $P_{x_{0}}^{2}\left(S_{0}^{b}<\infty\right)>0$ and therefore $P_{x_{0}}^{2}\left(\hat{S}_{0}^{b} \cap\left\{S_{0}^{b}<\infty\right\}\right)>0$. It is then easy to verify that $\hat{S}_{0}^{b} \cap\left\{S_{0}^{b}<\infty\right\}$ is a $\left(\pi_{1}, \pi_{2}, x_{0}\right)$ local singularity at $S_{0}^{b}$.

8.5. A remark on jump singularities. Let $J_{n}^{k}, n, k=1,2,3, \ldots$, be the stopping times defined in the proof of Theorem 5.1. Let $\mathscr{M}_{J_{n}^{k}}^{J_{k}}$ be the $\sigma$-field generated by the triple of random variables $\left(J_{n}^{k}, X_{J_{n}^{k}}, X_{J_{n}^{k}}^{\bar{k}}\right)$ and $\mathscr{M}_{\zeta}^{J}$ be the $\sigma$-field generated by the random variables $\zeta$ and $\chi_{\{\zeta<\infty\}} \cdot X_{\zeta}^{-}$. Then $\mathscr{M}_{J_{n}^{k}}^{J} \subset \mathscr{M}_{J_{n}^{k}}$ and $\mathscr{M}_{\zeta}^{J} \subset \mathscr{M}_{\zeta}$. We call the $\sigma$-fields $\mathscr{M}_{J_{n}^{k}}^{J_{k}}, k, n=1,2,3, \ldots$, and $\mathscr{M}_{\zeta}^{J}$ the jump $\sigma$-fields. An event $A$ is a $\left(\pi_{1}, \pi_{2}, x_{0}\right)$ jump field singularity if $A$ belongs to one of the jump $\sigma$-fields and is a $\left(\pi_{1}, \pi_{2}, x_{0}\right)$ jump singularity at the corresponding jump time. If the pair $\left(\pi_{1}, \pi_{2}\right)$ is either free of jump singularities or has jump field singularities then it is said to have the $J$-property. The pair $\left(\pi_{1}, \pi_{2}\right)$ of Example 3 of $\S 3$ has the $J$-property. However, the question of the characterization of pairs of processes which have the $J$-property is not considered in this paper.

A. V. Skorokhod [17] has considered the question of jump singularities for Markov processes which are defined by stochastic integral equations.

8.6. The decomposition theorem. Let $\pi_{1}$ and $\pi_{2}$ be a pair of Hunt processes which are instantaneously equivalent.

THEOREM 8.6.1. If $A_{0}$ is a $\left(\pi_{1}, \pi_{2}, x_{0}\right)$ singularity, then $A_{0} \subset C \cup D \cup G$ where $C$ is either empty or a tail type singularity, $D$ is either empty or a jump singularity and $G$ is either empty or a pseudo local singularity.

Proof. Given $A_{0}$ let $B_{0}^{\prime}$ be defined as in Proposition 6.5. Let $C \equiv B_{0}^{\prime} \cap\left\{T_{A_{0}}=\infty\right\}$. If $P_{x_{0}}^{2}(C)>0$, then for each $t \in[0, \infty), Y_{C}^{1}(t)=0, P_{x_{0}}^{2}$-almost surely (Proposition 6.3). Hence $C$ is a tail type singularity. Let $D \equiv B_{0}^{\prime} \cap\left\{T_{A_{0}}<\infty\right\} \cap\left\{X_{T_{A_{0}}} \neq X_{\bar{T}_{A_{0}}}\right\}$ and let $S$ be a stopping time such that $P_{x_{0}}^{2}\left(D \cap\left\{S<T_{A_{0}}\right\}\right)=P_{x_{0}}^{2}(D)$. Then Propositions 6.3 and 6.5 imply that $Y_{D}^{1}(S)=0, P_{x_{0}}^{2}$-almost surely on $D$. In addition, $T_{A_{0}}$ is $\pi_{2}$-totally inaccessible in the weak sense at $x_{0}$ on $D$ (Proposition 2.9.1). Hence $D$ is a $\left(\pi_{1}, \pi_{2}, x_{0}\right)$ jump singularity. Let $G \equiv B_{0}^{\prime} \cap\left\{T_{A_{0}}<\infty\right\} \cap\left\{X_{T_{A_{0}}}=X_{\bar{T}_{A_{0}}}\right\}$. Then Proposition 2.9.1 implies the existence of an increasing sequence of stopping times $\left\{T_{n}\right\}$ such that $\lim _{n \rightarrow \infty} T_{n}=T_{A_{0}}$ and $T_{n}<T_{A_{0}}<\infty$ for every $n$, both $P_{x_{0}}^{2}$-almost surely on $G$. Propositions 6.3 and 6.5 imply that for every $n, Y_{G}^{1}\left(T_{n}\right)=0, P_{x_{0}}^{2}$ almost surely on $G$. Hence $G$ is a pseudo local singularity and the proof is complete.

8.6. Continuous Hunt processes. Let $\pi_{1}$ and $\pi_{2}$ be a pair of continuous Hunt processes which are instantaneously equivalent. Then we have the following result.

THEOREM 8.7.1. (i) If $\pi_{1}$ and $\pi_{2}$ are free of local singularities then $\pi_{1}$ and $\pi_{2}$ are equivalent in finite time.

(ii) If in addition $\pi_{1}$ and $\pi_{2}$ are not strongly equivalent in finite time, then $\pi_{1}$ and $\pi_{2}$ must have a jump singularity at $\zeta$. 
(iii) If $\pi_{1}$ and $\pi_{2}$ are uninterrupted, then $\pi_{1}$ and $\pi_{2}$ are strongly equivalent in finite time.

Proof. (i) If $A_{0}$ is a $\left(\pi_{1}, \pi_{2}, x_{0}\right)$ or a $\left(\pi_{2}, \pi_{1}, x_{0}\right)$ singularity and $A_{0} \in \mathscr{F}_{t}$ for some $t \in[0, \infty)$ and $A_{0} \subset(\zeta>t)$, then $T_{A_{0}}<\zeta$ on $\left\{T_{A_{0}}<\infty\right\}$. But then $T_{A_{0}}$ is accessible on $A_{0}$ which in view of Theorem 8.4.1 implies the existence of a local singularity thus yielding a contradiction.

(ii) If $A_{0}$ is a $\left(\pi_{1}, \pi_{2}, x_{0}\right)$ or a $\left(\pi_{2}, \pi_{1}, x_{0}\right)$ singularity which belongs to $\mathscr{F}_{t}$ for some $t \in[0, \infty)$, then since $\pi_{1}$ and $\pi_{2}$ are free of local singularities $A$ must be a jump singularity. But if $\pi_{1}$ and $\pi_{2}$ are continuous, $J_{n}^{k}=+\infty, P_{x}^{i}$-almost surely, $i=1,2$, for each $x \in E$, every positive integer $k$ and every $n=1,2,3, \ldots$ Hence $A$ must be a jump singularity at $\zeta$.

(iii) If $\pi_{1}$ and $\pi_{2}$ are uninterrupted and continuous, then there can be no jump singularities. Hence if $\pi_{1}$ and $\pi_{2}$ are free of local singularities, then $\pi_{1}$ and $\pi_{2}$ are strongly equivalent in finite time.

8.8. Remarks on the examples of $\S 3$. Example 1 of $\S 3$ is an example of a pair of Markov processes which can have only tail field singularities. In Example 2 the pair $\left(\pi_{1}, \pi_{2}\right)$ have local singularities at the stopping times $T_{n}=n$, where $n$ is a positive integer. Note that in this case $\pi_{2}$ is not quasi left continuous and that the jumps occur at accessible times. Example 3 illustrates the phenomena of jump singularities. Although the processes in this example are not in the canonical form we have considered, the results that we have obtained are nevertheless applicable. As noted above the pair of processes in Example 3 has the $J$-property.

9. Locally smooth Markov processes. In this section we show that for a large class of Hunt processes the existence of local singularities implies the existence of germ field singularities.

Let $\pi=\left(\Omega, \mathscr{F}_{\infty},\left(P_{x}\right)_{x \in E_{\Delta}},\left(X_{t}\right)_{t \in[0, \infty]}\right)$ be a strong Markov process with respect to the $\sigma$-fields $\left(\tilde{\mathscr{M}}_{t}^{\pi}\right)_{t \in[0, \infty]}$. The Markov process $\pi$ is locally smooth if the following is satisfied. Let $x$ be any point in $E$; let $\left\{T_{n}\right\}$ be an increasing sequence of stopping times and $T$ be a stopping time such that $T_{n}<T$ for all $n$ and $\lim _{n \rightarrow \infty} T_{n}=T$, both $P_{x}$-almost surely on $\{T<\zeta\}$, and let $\left\{\Gamma_{n}\right\}$ be a sequence of $\mathscr{E}_{\Delta}$-measurable sets. Then for any positive integer $N$,

$$
P_{x}\left(X(T) \in C_{N}, \bigcap_{n=1}^{\infty}\left\{X\left(T_{n}\right) \in \Gamma_{n}\right\}\right)=0,
$$

where $C_{N} \equiv\left\{y: y \in E, P_{y}\left(T \cup_{n=N}^{\infty} \Gamma_{n}=0\right)=0\right\}$, and for $\Gamma \in \mathscr{E}_{\Delta}$,

$$
\begin{aligned}
T_{\Gamma} & \equiv \inf \left\{t: X_{t} \in \Gamma\right\} \\
& \equiv+\infty \quad \text { if the above set is empty. }
\end{aligned}
$$

(Note that it can be shown that $C_{N}$ is measurable with respect to the $\sigma$-field of universally measurable subsets of $E_{\Delta}$ (R. K. Getoor [8]).)

In $\S 10$ we introduce the fine topology connected with the Markov process. The 
property of local smoothness can be thought of as quasi left continuity in the fine topology. We begin by obtaining some preliminary results.

Proposition 9.1. Let $A_{n} \in \mathscr{M}_{T_{n}}, n=1,2,3, \ldots$, where $\left\{T_{n}\right\}$ is a decreasing sequence of stopping times such that $\lim _{n \rightarrow \infty} T_{n}=0, P_{x}$-almost surely for some $x \in E_{\Delta}$. If $A \equiv \bigcap_{k=1}^{\infty} \bigcup_{n=k}^{\infty} A_{n}$, then $A \in \mathscr{M}_{0}^{P}$, the completion of $\mathscr{M}_{0}$ with respect to the measure $P_{x}$.

Proof. It suffices to show that $A \in \mathscr{M}_{t}$ for each $t>0$. Since $A \in \mathscr{M}_{T_{n}}$ for each $n$, it follows that $A \cap\left\{T_{n} \leqq t\right\} \in \mathscr{M}_{t}$ for each $t>0$. Since

$$
A=\left(A \cap\left\{T_{n} \leqq t\right\}\right) \cup\left(A \cap\left\{T_{n}>t\right\}\right),
$$

it suffices to show that $A \cap \lim _{n \rightarrow \infty}\left\{T_{n}>t\right\} \in \mathscr{M}_{0}^{P}$. But

$$
A \cap \lim _{n \rightarrow \infty}\left\{T_{n}>t\right\} \subset\left\{\lim _{n \rightarrow \infty} T_{n}>0\right\} .
$$

Since $\left\{\lim _{n \rightarrow \infty} T_{n}>0\right\} \in \mathscr{M}_{0}$ and $P_{x}\left(\left\{\lim _{n \rightarrow \infty} T_{n}>0\right\}\right)=0$, therefore

$$
A \cap\left(\lim _{n \rightarrow \infty}\left\{T_{n}>t\right\}\right) \in \mathscr{M}_{0}^{P} .
$$

Proposition 9.2. Let $\left\{\Gamma_{n}\right\}$ be a sequence of compact subsets of $E$ such that for every positive integer $N$ and some $y \in E-\bigcup_{n=1}^{\infty} \Gamma_{n}, P_{y}\left(T_{\cup_{n=N}^{\infty} \Gamma_{n}}=0\right)=1$. Then for any $\varepsilon>0$ there exists a decreasing sequence of stopping times $\left\{T_{n}^{*}\right\}$ and an integral valued function $f(n), n \leqq f(n)<\infty$, such that

(i) $T_{n}^{*}>0$ for each $n$ and $\lim _{n \rightarrow \infty} T_{n}^{*}=0, P_{y}$-almost surely, and

(ii) $P_{y}\left(\bigcap_{n=1}^{\infty}\left\{X\left(T_{n}^{*}\right) \in \bigcup_{m=n}^{f(n)} \Gamma_{m}\right\}\right) \geqq 1-\varepsilon$.

Proof. For any integral valued function $f(n), n \leqq f(n)<\infty$, let

$$
\begin{aligned}
& T_{0}^{*} \equiv \infty \\
& T_{n}^{*} \equiv T_{\bigcup_{m=n \Gamma_{m}}^{f(n)}} \wedge 1 / n \wedge T_{n-1}^{*}, \quad n=1,2,3,4, \ldots
\end{aligned}
$$

Since $T_{\bigcup_{m=n}^{r} \Gamma_{m}} \downarrow 0, P_{y}$-almost surely as $r \rightarrow \infty$, then $P_{y}\left(X\left(T_{n}^{*}\right) \in \bigcup_{m=n}^{f(n)} \Gamma_{m}\right) \uparrow 1$ as $f(n) \rightarrow \infty$. Hence we can choose $f(n)$ so that $P_{y}\left(X\left(T_{n}^{*}\right) \in \bigcup_{m=n}^{f(n)} \Gamma_{m}\right)>1-\varepsilon / 2^{n}$. But then $P_{y}\left(\bigcap_{n=1}^{\infty}\left\{X\left(T_{n}^{*}\right) \in \bigcup_{m=n}^{f(n)} \Gamma_{m}\right\}\right) \geqq 1-\varepsilon$. The fact that $T_{n}^{*}>0$ for each $n, P_{y^{-}}$ almost surely follows from the right continuity of the paths.

COROLlaRY. There is a sequence of $\left(\mathscr{M}_{t}\right)$-stopping times $\left\{S_{n}\right\}$ satisfying (i) and (ii).

Proof. This follows immediately from Propositions 9.2 and 2.8.1.

Let $\pi_{1}$ and $\pi_{2}$ be a pair of instantaneously equivalent locally smooth Hunt processes. Then we have the following result.

THEOREM 9.1. The pair $\left(\pi_{1}, \pi_{2}\right)$ is free of local singularities except possibly at the stopping time $\zeta$.

Proof. Let us assume the contrary; in particular let $A_{0}$ be a $\left(\pi_{1}, \pi_{2}, x_{0}\right)$ local singularity at a stopping time $T$ with $T<\zeta, P_{x_{0}}^{2}$-almost surely on $A_{0}$. Then by the 
corollary to Proposition 8.2.1, $P_{x_{0}}^{2}\left(\left\{X\left(\lim _{n \rightarrow \infty} T_{n}\right) \in E\right\} \cap\left(\bigcap_{n=1}^{\infty}\left\{X\left(T_{n}\right) \in \widetilde{K}_{2 / n}^{b}\right\}\right)\right)>0$ for some $b>0$ and some increasing sequence of stopping times $\left\{T_{n}\right\}$, where the $\widetilde{K}_{2 / n}^{b}, n=1,2,3, \ldots$, are compact subsets of the sets $K_{2 / n}^{b}$. Since $\pi_{2}$ is locally smooth, there is a point $y \in E$ such that for each $N=1,2,3, \ldots$,

$$
P_{y}^{2}\left(T_{\cup_{m=N}^{\infty} \tilde{K}_{2 / m}^{b}}=0\right)=1 \text {. }
$$

Note that $y$ is a fine boundary point of each of the sets $K_{2 / m}^{b}$. We proceed to show that $y \in \bigcap_{t>0} K_{t}^{b}$ which contradicts the assumption that the processes are instantaneously equivalent (Proposition 7.2).

Since $\bigcap_{m=1}^{\infty} K_{2 / m}^{b}=\varnothing$ we can assume without loss of generality that $y \notin \bigcup_{m=1}^{\infty} \tilde{K}_{2 / m}^{b}$. Let $\left\{S_{n}\right\}$ be a sequence of $\left(\mathscr{M}_{t}\right)$-stopping times constructed as in the corollary to Proposition 9.2 such that for some integral-valued function $f(n)$,

$$
P_{y}^{2}\left(\bigcap_{n=1}^{\infty}\left\{X\left(S_{n}\right) \in \bigcup_{m=n}^{f(n)} \tilde{K}_{2 / m}^{b}\right\}\right) \geqq 1-\varepsilon
$$

for some $\varepsilon<b$. Then

$$
\begin{aligned}
& \theta_{S_{n}}^{-1}\left[\bigcup_{m=n}^{f(n)} \hat{K}_{2 / m}^{b}\right] \in \mathscr{M}_{S_{n}+2 / n} \quad \text { (Proposition 2.6.1), } \\
& P_{y}^{1}\left[\theta_{S_{n}}{ }^{-1}\left(\bigcup_{m=n}^{f(n)} \hat{K}_{2 / m}^{b}\right)\right]=0
\end{aligned}
$$

and

$$
P_{y}^{2}\left(\theta_{S_{n}}^{-1}\left(\bigcup_{m=n}^{f(n)} \hat{K}_{2 / m}^{b}\right)\right) \geqq b-\varepsilon .
$$

If $B \equiv \bigcap_{k=1}^{\infty} \bigcup_{n=k}^{\infty} \theta_{S_{n}}^{-1}\left(\bigcup_{m=n}^{f(n)} \hat{K}_{2 / m}^{b}\right)$ then $P_{y}^{1}(B)=0$ and $P_{y}^{2}(B) \geqq b-\varepsilon$. Furthermore, Proposition 9.1 implies that $B \in \mathscr{M}_{0^{y}}^{P_{2}^{2}}=\mathscr{M}_{0}^{P^{1}}$. Hence $y \in \bigcap_{t>0} K_{t}^{b}$, yielding a contradiction and the proof is complete.

COROLLARY. If $\left(\pi_{1}, \pi_{2}\right)$ are a pair of locally smooth and instantaneously equivalent continuous Hunt processes, then $\pi_{1}$ and $\pi_{2}$ are equivalent in finite time.

Proof. This follows immediately from Theorem 8.7.1(i) and Theorem 9.1.

REMARK 9.1. In view of Proposition 9.3 the equivalence class (for equivalence in finite time) of a locally smooth continuous Hunt process is described by the mapping

$$
\begin{gathered}
\Phi: E \rightarrow \Gamma_{0} \text { where } \Gamma_{0} \text { is the Stone space of } \mathscr{M}_{0}, \text { and } \\
\Phi(x) \equiv\left\{A: A \in \mathscr{M}_{0}, P_{x}(A)=0\right\} .
\end{gathered}
$$

It would be interesting if one could formulate some reasonable set of sufficient conditions in order that a mapping of the form (9.1) be associated with a continuous locally smooth Hunt process. If we call a continuous locally smooth Hunt process whose germ field probabilities are described by (9.1) a solution to the correspondence (9.1), then this is the problem of finding sufficient conditions for 
the existence of a solution. Since it is shown in Example 1 of $\S 3$ that $\Phi$ uniquely determines and is uniquely determined by the diffusive term of the infinitesimal generator (or stochastic differential equation) for a one-dimensional diffusion one might think of (9.1) as a generalized stochastic differential equation.

We next formulate a necessary condition for (9.1) to have a solution. Let $A_{i}$, $i=1,2,3, \ldots$, be sets of $\mathscr{M}_{0}$ such that for every $x \in E, P_{x}\left(A_{i}\right)=1$. Then if $\left\{r_{i}: i=1,2,3, \ldots\right\}$ is any countable dense subset of $[0, \varepsilon)$ for some $\varepsilon>0$, let $\hat{A}_{i} \equiv \theta_{r_{i}}^{-1} A_{i}$ and $\hat{A} \equiv \bigcup_{n=1}^{\infty} \bigcap_{\left\{i: r_{i} \leqq 1 / n\right\}} A_{i}$. Then $\hat{A} \in \mathscr{M}_{0}$ and a necessary condition for the existence of a solution to (9.1) is that for every $x \in E, P_{x}(\hat{A})=1$.

In general such a solution, if it exists, is not unique. In fact E. B. Dynkin [7] and $P$. Courrège [4] have systematically studied the construction of processes which are equivalent in finite time by means of multiplicative functionals. Another interesting problem is to formulate side conditions which would pick out a unique solution to (9.1). In the case of stochastic differential equations for uninterrupted diffusions on the real line (cf. $\S 3$, Example 1) this is done by prescribing the drift term of the stochastic differential equation, that is, the limit:

$$
\lim _{t \downarrow 0} E_{x}((X(t)-x) / t) \equiv a(x)
$$

10. Characterization of locally smooth processes. In $\S 9$ it was shown that equivalence in finite time is equivalent to instantaneous equivalence for the class of continuous locally smooth Hunt processes. It turns out that the definition of local smoothness is intimately connected with certain concepts in probabilistic potential theory. In this section we discuss these connections. We begin by reviewing a few ideas from probabilistic potential theory. The reader is referred to G. A. Hunt [10], E. B. Dynkin [7] or R. K. Getoor [8] for more details. In this section $\pi=\left(\Omega, \mathscr{F}_{\infty},\left(P_{x}\right)_{x \in E_{\Delta}},\left(X_{t}\right)_{t \in[0, \infty]}\right)$ is a Hunt process which is considered as a strong Markov process with respect to the $\sigma$-fields $\left(\tilde{\mathscr{M}}_{t}^{\pi}\right)_{t \in[0, \infty]}$.

If $\Gamma \subset E_{\Delta}$, then $x$ is irregular for $\Gamma$ if there exists a Borel set $\Gamma^{\prime} \supset \Gamma$ such that $P_{x}\left(T_{\Gamma^{\prime}}>0\right)=1$. If $x$ is not irregular for $\Gamma$, then it is said to be regular for $\Gamma$. Let $\Gamma^{r}$ denote the set of regular points of $\Gamma$. A set $\Gamma$ is open in the fine topology if each $x \in \Gamma$ is irregular for $\Gamma^{C}$. A set $\Gamma \subset E_{\Delta}$ is said to be nearly analytic if for each $\mu$ there are analytic sets $\Gamma_{1}$ and $\Gamma_{2}$ such that $\Gamma_{1} \subset \Gamma \subset \Gamma_{2}$ and $P_{\mu}\left(X_{t} \in \Gamma_{2}-\Gamma_{1}\right.$ for some $t \geqq 0$ ) $=0$. A set $\Gamma$ is thin if it is contained in a nearly analytic set $\Gamma^{\prime}$ which has no regular points. A set is semipolar if it can be covered by a countable union of thin sets. Note that if $\Gamma \in \mathscr{E}$ then it can be shown that $\Gamma-\Gamma^{r}$ is semipolar. A set $\Gamma$ is polar if it is contained in a nearly analytic set $\Gamma^{\prime}$ such that $P_{x}\left(T_{\Gamma^{\prime}}<\infty\right)=0$ for each $x \in E$. Note that every polar set is semipolar.

A nonnegative function $f$ on $E_{\Delta}$ with $f(\Delta)=0$ which is measurable with respect to the $\sigma$-field of universally measurable sets is said to be excessive if $P_{t} f \leqq f$ for each $t \geqq 0$ and $P_{t} f \rightarrow f$ as $t \rightarrow 0$ pointwise. In particular the constant functions are excessive for any Hunt process having a Feller semigroup. 
Proposition 10.1. (i) If $f$ is excessive, the function $t \leadsto f\left(X_{t}\right)$ is right continuous and finite for all $t \geqq s$ if $f\left(X_{s}\right)$ is finite, $P_{x}$-almost surely for each $x \in E$.

(ii) For each $x \in E, f\left(X_{s}\right)$ is a $P_{x}$-supermartingale.

(iii) An excessive function is continuous in the fine topology.

Proof. Refer to E. B. Dynkin [7].

An excessive function $f$ is regular if $t \leadsto f\left(X_{t}\right)$ is continuous whenever $t \leadsto X_{t}$ is continuous, $P_{x}$-almost surely for each $x \in E$. A function $f$ is said to be a harmonic function if both $f$ and $-f$ are excessive.

Proposition 10.2. If there exists an excessive function $f$ which is not regular, then the Markov process is not locally smooth.

Proof. For $\varepsilon>0$, let $T^{\varepsilon} \equiv \inf \left\{t:\left|f\left(X_{t}\right)-f^{-}\left(X_{t}\right)\right|>\varepsilon, X_{t}=X_{t}^{-}\right\}$, where

$$
f^{-}\left(X_{t}\right) \equiv \lim _{s \uparrow t} f\left(X_{s}\right) \text {. }
$$

(Recall that $f\left(X_{t}\right)$ is free of oscillatory discontinuities, $P_{x_{0}}$-almost surely for each $x_{0} \in E$ (P. A. Meyer [13, Chapter 6, T 3]).) If $f$ is a nonregular excessive function, then for some $\varepsilon_{0}>0$ and $x_{0} \in E, P_{x_{0}}\left(T^{\varepsilon_{0}}<\infty\right)>0$. But then for some positive integer $k$,

$$
P_{x_{0}}\left(\left|f\left(X_{T^{\varepsilon_{0}}}\right)-f^{-}\left(X_{T^{\varepsilon_{0}}}\right)\right|>\varepsilon_{0}, k \varepsilon_{0} / 4 \leqq f\left(X_{T^{\varepsilon_{0}}}\right)<(k+1) \varepsilon_{0} / 4\right)>0 .
$$

Let

$$
A_{\varepsilon_{0}} \equiv\left\{\left|f\left(X_{T^{\varepsilon_{0}}}\right)-f^{-}\left(X_{T^{\varepsilon_{0}}}\right)\right|>\varepsilon_{0}, k \varepsilon_{0} / 4 \leqq f\left(X_{T^{\varepsilon_{0}}}\right)<(k+1) \varepsilon_{0} / 4\right\} .
$$

Let $\left\{T_{n}\right\}$ be an increasing sequence of stopping times such that $T_{n}<T^{\varepsilon_{0}}$ for all $n$ and $\lim _{n \rightarrow \infty} T_{n}=T^{\varepsilon_{0}}$, both $P_{x_{0}}$-almost surely on $A_{\varepsilon_{0}}$, and let

and

$$
\Gamma \equiv\left\{x: f(x) \notin\left[(k-1) \varepsilon_{0} / 4,(k+2) \varepsilon_{0} / 4\right]\right\}
$$

$$
\Gamma^{\prime} \equiv\left\{x: f(x) \in\left[k \varepsilon_{0} / 4,(k+1) \varepsilon_{0} / 4\right]\right\} .
$$

Then $\Gamma^{\prime} \subset\left\{x: P_{x}\left(T_{\Gamma}=0\right)=0\right\}$ because of the fine continuity of $f$. Since $P_{x_{0}}\left(A_{\varepsilon_{0}}\right)>0$, there is a positive integer $N$ such that $P_{x_{0}}\left(\bigcap_{n=N}^{\infty} X\left(T_{n}\right) \in \Gamma, X(T) \in \Gamma^{\prime}\right)>0$. Hence the process is not locally smooth.

REMARK 10.1. It can also be proved that if there is a semipolar set which is not polar, then the process is not locally smooth. However we omit the proof.

Let $\Gamma \subset E$ and let $f$ be an excessive function. If $R_{f}^{\Gamma} \equiv \inf \{u: u$ excessive, $u \geqq f$ on $\Gamma$, then $R_{f}^{\Gamma}$ is called the réduite of $f$ on $\Gamma$. We make the following assumptions concerning the réduite.

Assumption B. (i) There is a unique excessive function, $\hat{R}_{f}^{\Gamma}$, which differs from $R_{f}^{\Gamma}$ on a semipolar set. $\hat{R}_{f}^{\Gamma}$ is called the balayage of $f$ on $\Gamma$.

(ii) If $y \notin \Gamma$ is irregular for $\Gamma$, then there is a neighborhood $N_{y}$ of $y$ such that $R_{1}^{\Gamma \cap N_{y}}(y)<1$.

(iii) The set $\left\{y: y \in \Gamma, \hat{R}_{1}^{\Gamma} \neq 1\right\}$ is semipolar. 
RemarK 10.2. M. Brelot [2] has shown that Assumption B is valid if the harmonic functions satisfy a certain set of axioms. In particular these axioms are satisfied if the infinitesimal generator is an elliptic differential operator with smooth coefficients. In the general probabilistic context, the balayage and réduite are discussed in the forthcoming book of R. M. Blumenthal and R. K. Getoor [3].

THEOREM 10.1. If Assumption $\mathrm{B}$ is satisfied and

(10.1) every semipolar set is polar, and

(10.2) every finite excessive function is regular, then the Markov process is locally smooth.

Proof. Let us show that if $\pi$ is not locally smooth and (10.1) is satisfied, then (10.2) is not satisfied. If $\pi$ is not locally smooth then there is a sequence of stopping times $\left\{T_{n}\right\}$, a stopping time $T$, a sequence $\left\{\Gamma_{n}\right\}$ of $\mathscr{E}_{\Delta}$-measurable sets and a point $x \in E$ such that:

(i) $T_{n}<T$ for all $n$ and $\lim _{n \rightarrow \infty} T_{n}=T$, both $P_{x}$-almost surely on $\{T<\zeta\}$, and

(ii) for some positive integer $N$,

$$
P_{x}\left(\bigcap_{n=N}^{\infty}\left\{X\left(T_{n}\right) \in \Gamma_{n}\right\}, X(T) \in C_{N}\right)>0,
$$

where $C_{N} \equiv\left\{y: y \in E, P_{y}\left(T_{K_{N}}=0\right)=0\right\}$ and $K_{N} \equiv \bigcup_{n=N}^{\infty} \Gamma_{n}, N=1,2,3, \ldots$ As noted above $\left(C_{N} \cap K_{N}\right)$ is semipolar and hence by (10.1) is polar. If $y \in C_{N}-K_{N}$, then $y$ is an irregular point for $K_{N}$. Assumption B implies that there is a neighborhood $\Lambda_{i}$ of $y\left(\Lambda_{i}\right.$ is assumed to belong to a countable base for the open sets of $E$, say $\left.\left\{\Lambda_{i}\right\}\right)$ for which $R_{1}^{K_{N} \cap \Lambda_{i}}(y)<1$. In other words

$$
y \in \Sigma_{\mathfrak{i}} \equiv\left\{y: R_{1}^{K_{N} \cap \Lambda_{i}}(y)<1\right\} \cap \Lambda_{i} .
$$

But then $C_{N}-K_{N} \subset \bigcup_{i=1}^{\infty} \Sigma_{i}$ where the union runs over the countable base $\left\{\Lambda_{i}\right\}$. Let $\hat{\Sigma}_{i} \equiv\left\{y: \hat{R}_{1}^{K_{N} \cap \Lambda_{i}}(y)<1\right\} \cap \Lambda_{i}$. Since $\hat{R}_{1}^{K_{N} \cap \Lambda_{i}}=R_{1}^{K_{N} \cap \Lambda_{i}}$ except on a semipolar and hence by (10.1) a polar set, there is some $i_{0}$ such that

$$
P_{x}\left(\bigcap_{n=N}^{\infty}\left\{X\left(T_{n}\right) \in \Gamma_{n}\right\}, X(T) \in \hat{\Sigma}_{i_{0}}\right)>0 .
$$

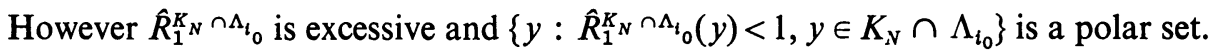
Therefore since $X_{T}=X_{T}^{-}, P_{x^{-}}$-almost surely on $\{T<\zeta\}$ (cf. Proposition 2.9.1), $\hat{R}_{1}^{K_{N} \cap \Lambda_{i_{0}}}(X(t))$ is discontinuous at $T, P_{x}$-almost surely on the set $\left(\bigcap_{n=N}^{\infty}\left\{X\left(T_{n}\right) \in \Gamma_{n}\right\}\right.$, $X(T) \in \hat{\Sigma}_{i_{0}}$ ) which has positive $P_{x}$-probability. But then (10.2) is not satisfied and the proof is complete.

REMARK 10.3. A discussion of (10.1) and (10.2) and their interrelation is given in G. A. Hunt [10] and R. K. Getoor [8].

J. L. Doob [6] has shown that the harmonic functions and excessive functions of the Wiener process in a Euclidean space are the harmonic and superharmonic 
functions, respectively, of classical potential theory. However the classical superharmonic and harmonic functions are such that Assumption B as well as (10.1) and (10.2) are satisfied (refer to M. Brelot [1] and E. B. Dynkin [7]). Hence the Wiener process is locally smooth.

\section{REFERENCES}

1. M. Brelot, Eléments de la théorie classique du potential, 3rd ed., Centre de Documentation Universitaire, Paris, 1965.

2. - Lectures on potential theory, Collection of the Tata Institute No. 19, Bombay, 1960.

3. R. M. Blumenthal and R. K. Getoor, Markov processes and potential theory, (to appear).

4. P. Courrège, Fonctionnelles multiplicatives, sous-processus d'un processes de Markov et semi-groupes subordonnés, Séminaire de théorie du potentiel (Brelot-Choquet-Deny), Sixth year, 1961-1962.

5. P. Courrège and P. Priouret, Temps d'arrêt d'une fonction aléatoire: relations d'équivalence associées et propriétés de décomposition, Publ. Inst. Statist. Univ. Paris 14 (1965), 245-274.

6. J. L. Doob, Semimartingales and subharmonic functions, Trans. Amer. Math. Soc. 77 (1954), 86-121.

7. E. B. Dynkin, Markov processes, Academic Press, New York, 1965.

8. R. K. Getoor, Additive functionals and excessive functions, Ann. Math. Statist. 36 (1965), 409-422.

9. P. R. Halmos, Measure theory, Van Nostrand, Princeton, N. J., 1950.

10. G. A. Hunt, Markoff processes and potentials, Illinois J. Math. 1 (1957), 42-93, 2 (1958), 151-213.

11. K. Itô and H. P. McKean, Diffusion processes and their sample paths, Academic Press, New York, 1965.

12. P. A. Meyer, Decomposition of supermartingales; The uniqueness theorem, Illinois J. Math. 7 (1963), 1-17.

13. — Probability and potentials, Blaisdell, Waltham, Mass., 1966.

14. J. Neveu, Bases mathématiques du calcul des probabilités, Masson, Paris, 1964.

15. L. A. Shepp, Radon-Nikodym derivatives of Gaussian measures, Ann. Math. Statist. 37 (1966), 321-354.

16. A. V. Skorokhod, On the differentiability of measures which correspond to stochastic processes, Theor. Probability Appl. 5 (1960), 40-49.

17. - Studies in the theory of random processes, Addison-Wesley, Reading, Mass., 1965.

MCGILL UNIVERSITY, Montreal, Canada 